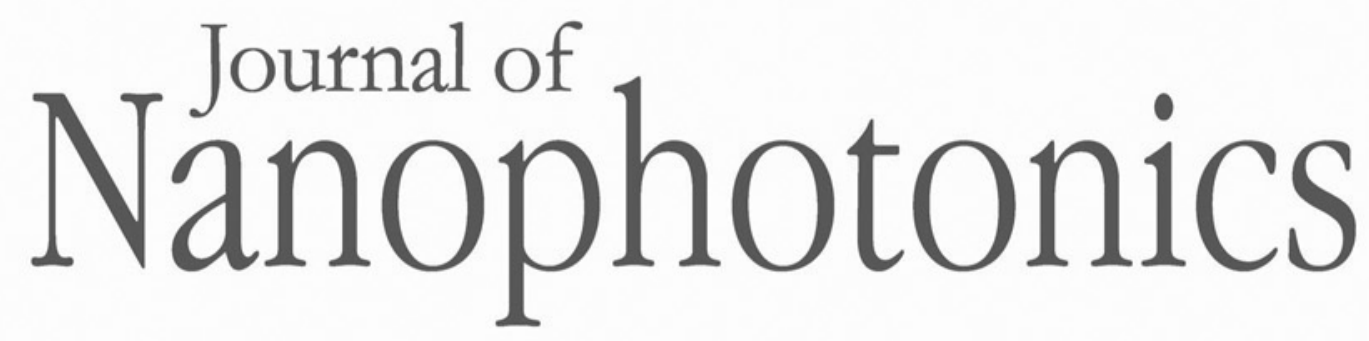

Nanophotonics.SPIEDigitalLibrary.org

\title{
Superluminality phenomenon at a femtosecond laser pulse propagation in a medium containing nanorods
}

Vyacheslav A. Trofimov

Tatiana M. Lysak 


\title{
Superluminality phenomenon at a femtosecond laser pulse propagation in a medium containing nanorods
}

\author{
Vyacheslav A. Trofimov* and Tatiana M. Lysak \\ Lomonosov Moscow State University, Moscow, Russia
}

\begin{abstract}
We show a possibility for soliton formation and superluminality phenomenon at a femtosecond pulse propagation in a medium with noble nanorods. These effects take place if a positive phase grating is induced by laser radiation. We take into account the dependence of two- or one-photon absorption [single-photon absorption (SPA)] on the nanorod aspect ratio and time-dependent nanorod aspect ratio changing due to nanorods reshaping (or melting) because of laser energy absorption. We demonstrate that a fast light propagation mode occurs for various detuning between wave packet carrier frequency and nanorod resonance frequency, which is a key parameter for practical observation of fast or slow light in a physical experiment. We also developed analytical approaches for explanation of laser pulse propagation peculiarities in a medium with nanorods. In particular, in the framework of nonlinear geometric optics approximation, we derived the laws for the pulse intensity and instantaneous frequency evolution if a phase grating is induced by laser radiation in a medium with SPA. We also developed an approximate analytical soliton and derived the chirped soliton amplitude, duration and homogeneous shift evolution, carrier frequency changing, and pulse chirp evolution. The results of analytical consideration are confirmed by computer simulation results. (c) The Authors. Published by SPIE under a Creative Commons Attribution 3.0 Unported License. Distribution or reproduction of this work in whole or in part requires full attribution of the original publication, including its DOI. [DOI: 10 .1117/1.JNP.11.026003]
\end{abstract}

Keywords: femtosecond pulses; superluminality; chirped solitons; nanorods; phase-amplitude grating; aspect ratio.

Paper 16174 received Nov. 11, 2016; accepted for publication Apr. 5, 2017; published online Apr. 25, 2017.

\section{Introduction}

In recent years, thin films, doped with noble (gold or silver) metal nanoparticles, have attracted attention as recording media due to their strong nonlinear response resonance to the frequency of incident optical radiation and electric field polarization, and nanoparticles aspect ratio changing because of photothermal melting or reshaping. ${ }^{1-6} \mathrm{~A}$ large number of investigations deal with the optical response of a large ensemble of metal nanoparticles embedded into a medium and focus their attention on nonlinear refractive index changing and the third-order susceptibility changing in dependence of a nanoparticles concentration. ${ }^{7-12}$ Obviously, a clarification of the physical mechanism for nanoparticle optical response dependence on the nanorods aspect ratio and nanorods orientation, and local enviroment, ${ }^{13-18}$ as well as photothermal reshaping of nanorods or wires at the temperatures less than the bulk melting temperature ${ }^{19-22}$ are very important questions. Various mathematical and physical models for an explanation of photothermal reshaping of nanosized nanoparticles are proposed up to now. ${ }^{19,20,23-25}$

One of the possible applications of nanorods is their use in data storage devices. ${ }^{1-6}$ For this problem, a self-similar mode of laser pulse propagation is of great importance. This is due to the fact that the laser radiation spectrum distortions, caused by nonlinear refraction and nonlinear absorption, can induce false recording or writing of information because these processes are very sensitive to the pulse spectrum. Indeed, nanoparticles reshaping leads to the laser pulse spectrum changing caused by the pulse chirping and laser energy absorption. Consequently,

*Address all correspondence to: Vyacheslav A. Trofimov, E-mail: vatro@cs.msu.ru 
false information writing may take place. Therefore, a mode of laser pulse propagation with unchanging spectrum is preferable for data processing based on nanorods using.

As it is well-known, a self-similar or soliton mode of laser pulse propagation in homogeneous and layered media is a subject of numerous studies in recent years. ${ }^{26-34}$ However, such a laser pulse propagation mode for a chirped pulse has not been investigated enough yet, despite its wide appearance in a high intense femtosecond pulse propagation. In our previous papers, ${ }^{35-38}$ we have investigated a femtosecond pulse propagation in a medium with nanorods under the conditions of both the nanorod ellipticity (aspect ratio) changing and dependence of two-photon absorption (TPA) on nanoparticle aspect ratio. In particular, using the density matrix formalism, we derived the equation set, which describes the femtosecond pulse propagation in the medium with nanorods reshaping, and obtained some analytical formulas for such propagation. ${ }^{37}$ In particular, we analytically derived the laws for laser pulse energy and intensity evolution if the laser pulse propagates in a medium with TPA or single-photon absorption (SPA) under the conditions of negligible second-order dispersion (SOD) influence. We also derived the laws for laser pulse amplitude and aspect ratio evolution in the framework of nonlinear geometric optics approximation if only a pure amplitude grating is induced by laser radiation in the medium with SPA. Note that the formulas for laser pulse amplitude evolution, obtained in different approaches, coincide for a small propagation distance depending on the propagation parameters. These laws allowed us to discuss the influence of the incident pulse chirp on the pulse center shift in the area of time increasing or decreasing. Earlier, we investigated the self-similar mode of laser pulse propagation in homogeneous media with TPA or multiphoton absorption (MPA) and showed that the appropriate initial frequency chirp is crucial for the self-similar mode realization. ${ }^{39,40}$ In Ref. 38, we also discussed, in detail, the pulse chirp role and described a nonlinear chirp formation due to the light energy absorption and the positive phase-amplitude grating in the media with nanorods. Investigating the influence of the relation between the nanorods absorption spectrum bandwidth and laser pulse spectrum bandwidth on laser pulse spectrum distortion, we found out that the laser pulse spectrum distortion is really absent under certain conditions despite the nonlinearity action. ${ }^{36}$

Our attention in this paper is attracted by the effects of superliminality, ${ }^{41-46}$ as well as a soliton formation. It should be stressed that earlier ${ }^{37}$ we showed that a fast light can appear in a medium with nanorods under the weak optical energy absorption on the pulse dispersion length while below, we show that the laser pulse propagation modes take place for a wide range of the problem parameters. In particular, we demonstrate that a fast light propagation mode occurs for various strengths of positive phase-amplitude grating induced by laser radiation, which correspond to the various detuning between wave packet carrier frequency and nanorod resonance frequency. Because this parameter is key for a practical observation of fast light in a physical experiment, we consider its sufficient small value. We also investigate the soliton formation if a nonlinear absorption is close to the maximal value, which is achieved at absorption resonance of nanorods in dependence on their aspect ratio. In connection with this, it should be stressed that, in our previous papers, ${ }^{35-38}$ we considered a linear dependence of light energy absorption on nanorod aspect ratio, which is valid far from the absorption resonance.

We derived the laws for the laser pulse energy, intensity, carrier frequency of a wave packet, and a pulse chirp in the framework of nonlinear geometric optics approximation. However, we consider laser pulse propagation in a medium with SPA under the conditions of inducing phase-amplitude grating. We compared the results of analytical consideration with the computer simulation results and formulated conditions of nonlinear geometric optics approximation validity for the problem under consideration. To prove our computer simulation results, we developed the approximate analytical soliton, which allows us to write the soliton amplitude, duration, and phase evolution along propagation coordinate.

\section{Problem Statement}

We describe a femtosecond laser pulse propagation in a medium with nanorods (Fig. 1) in the framework of a semiclassical approach. Therefore, we describe the atomic states using a density matrix formalism while the electric field strength is described classically in the framework of a slowly varying envelope. Due to a nanorod strong nonlinear response resonance to the incident 


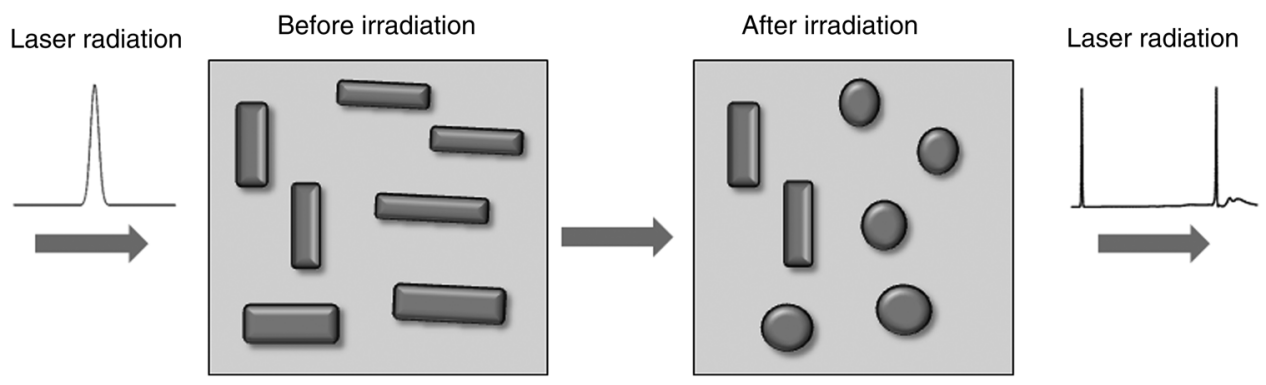

Fig. 1 Schematic showing of laser radiation interaction with the medium containing nanorods.

optical radiation frequency, we restrict our consideration by the two-level medium and take into account the dependence of light absorption on a nanorod aspect ratio. Thus, a femtosecond laser pulse propagation along the $z$-axis can be described in the framework of slowly varying envelope $E(z, t)$ of wave packet for electric field strength $\mathrm{E}(z, t)$ by the following set of equations: $:^{47}$

$$
\begin{gathered}
\frac{\partial E}{\partial z}+\frac{1}{v_{g}} \frac{\partial E}{\partial t}+i D_{2} \frac{\partial^{2} E}{\partial t^{2}}=i \frac{2 \pi \hbar \omega_{p}^{2}}{c^{2} k_{p}} N_{s}\left[\frac{\chi_{1}-\chi_{2}}{2 \hbar}\left(N-N_{0}\right) E+k r_{12} \rho_{12} E^{*(k-1)}\right], \\
\frac{\partial \rho_{12}}{\partial t}+\left[\frac{1}{T_{\perp}}-i\left(\nu-\frac{\chi_{1}-\chi_{2}}{\hbar}|E|^{2}\right)\right] \rho_{12}=i r_{12} f(\varepsilon) E^{k} N, \\
\frac{\partial N}{\partial t}+\frac{1}{T_{\|}}\left[N-N_{0}\right]=-4 r_{12} \operatorname{Im}\left(E^{* k} \rho_{12}\right), \quad k=1,2, \quad z>0, \quad 0<t<L_{t},
\end{gathered}
$$

without taking into account its diffraction and with initial and boundary conditions

$$
E(z=0, t)=E_{0}(t), \quad E(z, t=0)=E\left(z, t=L_{t}\right)=0, \quad \rho_{12}(z, t=0)=0, \quad N(z, t=0)=N_{0},
$$

where $E(z, t)$ is related with the electric field strength by relation

$$
\mathrm{E}(z, t)=\frac{1}{2}\left\{E(z, t) \exp \left[-i\left(\omega_{p} t-k_{p} z\right)\right]+\text { c.c. }\right\},
$$

where $\omega_{p}$ is a frequency and $k_{p}$ is a wavenumber of the laser pulse; $v_{g}=\frac{\partial \omega_{p}}{\partial k_{p}}$ is a group velocity and $D_{2}=\frac{1}{2} \frac{\partial^{2} k_{p}}{\partial \omega_{p}^{2}}$ is the SOD of a medium; $N=\rho_{11}-\rho_{22}$ is an inversion or population difference of energy levels 1 and 2; $\rho_{11}$ and $\rho_{22}$ are populations of the first and the second energy level of an atom, respectively; $N_{0}$ is an equilibrium value of the inversion corresponding to laser radiation absence $(E=0)$ and its value varies from -1 to $1,{ }^{47} N_{0}=1$ corresponds to passive medium and the value $N_{0}=-1$ corresponds to active medium; $\rho_{12}$ is a nondiagonal element of the density matrix; $\omega_{21}$ is the frequency of transition between the energy levels 2 and $1 ; \nu=2 \omega_{p}-\omega_{21}$ is a frequency detuning between the two photons of laser radiation and the energy level transition frequency; $T_{\|}$and $T_{\perp}$ are longitudinal and transverse relaxation times, respectively; $N_{s}$ is the density of atoms; $\chi_{1,2}$ are linear polarizabilities of the medium with atoms in the energy states 1 and 2 , respectively, at the frequency $\omega_{p}$

$$
r_{12}=\left\{\begin{array}{ll}
\hbar^{-1}\left|d_{21}\right|, & \text { one-photon absorption }(k=1) \\
\hbar^{-2} \sum_{n} d_{1 n} d_{n 2} /\left(\omega_{n 1}-\omega_{p}\right), & \operatorname{TPA}(k=2)
\end{array},\right.
$$

where $d_{i j}$ is the matrix elements of the dipole moment, $k$ is the number of photons involved in the absorption process: $k=1$ corresponds to SPA, $k=2-$ TPA, $c$ is a light velocity, $\hbar$ is the Planck's constant, and $L_{t}$ is a time interval during which we analyze laser pulse interaction with 


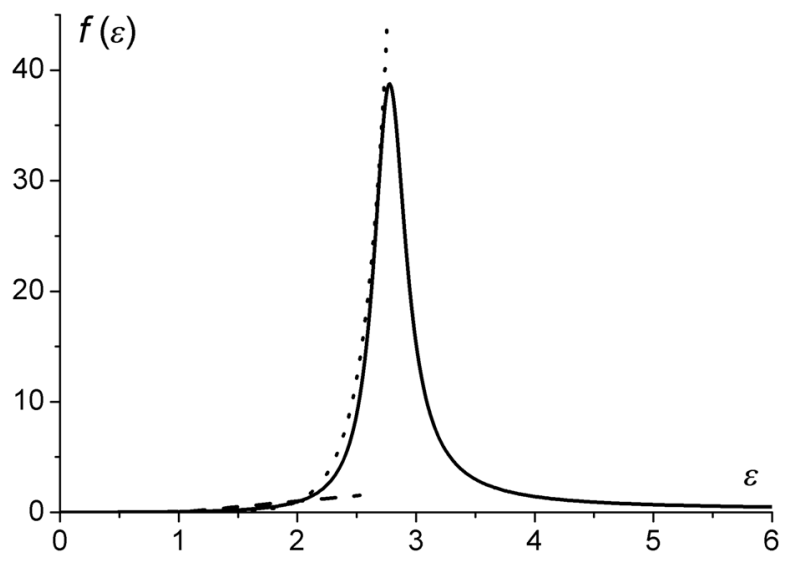

Fig. 2 Dependence $f(\varepsilon)$ defined by Eqs. (6) and (7) for gold nanorods at the falling radiation with $\lambda=800 \mathrm{~nm}$ (solid line), or by Eq. (4) (dashed line), or by Eq. (5) (dotted line).

a medium. Ellipticity (aspect ratio) $\varepsilon$ of nanorods is determined as $\varepsilon=L / W$, where $W$ is the width and $L$ is the length of a nanorod, or equivalently, as $\varepsilon=a / b$, where $a$ and $b$ are the major and minor axes of an ellipsoidal nanorod. As a rule, the aspect ratio of nanorods used for data recording varies from 1.5 to $9 .{ }^{18,48}$ However, in physical experiments, a nanorod shape transform to a sphere also realizes. The function $f(\varepsilon)$ describes the dependence of TPA or SPA process on the nanorod aspect ratio $\varepsilon$.

Using the papers, ${ }^{5,18,47,48}$ we can write some physical parameters, listed above: the gold atoms density belongs to the interval $N_{s}=1.1 \cdot 10^{17}-8.6 \cdot 10^{18} \mathrm{~cm}^{-3}$ (molar concentration of gold nanoparticles varies from $979 \mathrm{pM}$ to $40 \mathrm{nM}$ ), ${ }^{5,48}$ the incident laser radiation wavelength is $\lambda=800 \mathrm{~nm}$; the pulse duration is ${ }^{1-4} 100$ fs to $6 \mathrm{~ns} ;\left|r_{12}\right|=10^{3} \mathrm{~cm} \cdot \mathrm{s} / \mathrm{g}$, the longitudinal and transverse relaxation times are less than $T_{\|}=10^{-12} \mathrm{~s}$ and $T_{\perp}=10^{-13} \mathrm{s.}^{47}$ The difference of linear polarizabilities is $\chi_{2}-\chi_{1} \approx 10^{-25} \mathrm{~cm}^{3}$.

We consider three possible types of the dependence $f(\varepsilon)$ on nanorod aspect ratio

$$
\begin{gathered}
f(\varepsilon)=\varepsilon-1, \\
f(\varepsilon)=\exp [5(\varepsilon-2)], \quad \varepsilon \leq 2.7 \\
f(\varepsilon)=\frac{\left(\varepsilon_{2} / A^{2}\right)}{\left(\varepsilon_{1}+\frac{1-A}{A} \varepsilon_{m}\right)^{2}+\varepsilon_{2}^{2}}, \\
A=\left[1-\frac{\xi Q_{1}^{\prime}(\xi)}{Q_{1}(\xi)}\right]^{-1}, \xi=\left[1-\left(\frac{1}{\varepsilon}\right)^{2}\right]^{-1 / 2}, Q_{1}(\xi)=\left(\frac{\xi}{2}\right) \ln \left[\frac{\xi+1}{\xi-1}\right]-1, Q_{1}^{\prime}(\xi)=\frac{\mathrm{d} Q_{1}(\xi)}{\mathrm{d} \xi} .
\end{gathered}
$$

The dependence [Eqs. (6) and (7)] follows from Boyd et al. ${ }^{49}$ shape factor expression for the absorption coefficient of gold nanorods, calculated in the dipole approximation, ${ }^{50,51}$ where $\varepsilon_{m}$ is the dielectric permittivity of the ambient medium, $\varepsilon_{1}+i \varepsilon_{2}$ is the complex dielectric permittivity of the nanorod. It should be stressed that the dependence $f(\varepsilon)$ in Eq. (4) adequately approximates the dependence [Eqs. (6) and (7)] for physical parameters $\left(\varepsilon_{1}=-22.1, \varepsilon_{2}=1.8\right.$ for Au at $\lambda=800 \mathrm{~nm}^{52}$ and $\varepsilon_{m}=3$ ) if the aspect ratio varies from 1 to 2 (Fig. 2, dashed line), whereas the dependence $f(\varepsilon)$ in Eq. (5) is valid if the aspect ratio changes from 1 to 2.6 (Fig. 2, dotted line). Obviously, the second dependence is more preferable for a laser pulse interaction with nanorods near the nonlinear absorption resonance. Below, we use both dependences.

The aspect ratio change due to the nanorods reshaping can be described by the following equation: 


$$
\frac{\partial \varepsilon}{\partial t}+\hat{\varsigma} \frac{1}{T_{\|}}\left(N_{0}-N\right)=0
$$

The parameter $\hat{\varsigma}$ characterizes the energy releasing due to the nonradiative relaxation of the excited energy levels. This part of releasing energy thermalizes and is spent on the nanorods reshaping. The other part of absorbed energy may be spent on the radiative energy transitions, corresponding to luminescence, for example. ${ }^{53}$ Of course, there are other mechanisms of exited energy levels relaxation. Let us note that one can show that the second term in Eq. (8) depends on the function $f(\varepsilon)$ if a quasistationary case of a laser pulse interaction with the nanorods occurs (see below).

Initial condition for the nanorod aspect ratio is

$$
\varepsilon(z, t=0)=\varepsilon_{0}, \quad 0 \leq z \leq L_{z},
$$

where $L_{z}$ is the length of a medium containing nanorods. Below, we choose the initial value of aspect ratio $\varepsilon_{0}=2$ for the dependence of Eq. (4) and $\varepsilon_{0}=2.6$ for the dependence of Eq. (5).

To simplify Eqs. (1)-(3) by omitting the laser energy absorption dynamics, let us suppose that the pulse duration is much greater than $T_{\|}$and $T_{\perp}$. So, we consider the pulses with duration more than 500 fs. Such pulses are widely used in practice. ${ }^{3,4}$ Then, Eqs. (2) and (3) become stationary, and it is possible to express the medium inversion $N$ and the density matrix nondiagonal element $\rho_{12}$ from these equations as functions of the electric field slowly varying envelope $E(z, t)$ and $f(\varepsilon)$. We also believe that the energy level transition saturation is absent $\left[m_{12} f(\varepsilon)|E|^{4} \ll 1\right]$, and the difference between linear polarizabilities $\chi_{1}$ and $\chi_{2}(p=0)$ can be neglected. In this case, the following set of dimensionless quasistationary equations for the slowly varying amplitude of laser pulse and the aspect ratio evolution can be derived for the system of coordinates that moves with the light pulse velocity ${ }^{37}$

$$
\begin{gathered}
\frac{\partial A}{\partial z}+i D \frac{\partial^{2} A}{\partial t^{2}}+f(\varepsilon)\left(\delta_{0}+i \xi\right)|A|^{2(k-1)} A=0, \\
\frac{\partial \varepsilon}{\partial t}=-\tilde{\delta} f(\varepsilon)|A|^{2 k},
\end{gathered}
$$

where

$$
A(z, t)=E(z, t) \exp [-i(\beta t+\gamma z)] / A_{0}, \quad \beta=\frac{1}{v_{g}} \frac{\tau_{p}}{2 D_{2}}, \quad \gamma=\left(\frac{1}{v_{g}}\right)^{2} \frac{l_{\mathrm{dis}}}{4 D_{2}}
$$

is a dimensionless complex amplitude of laser radiation, normalized on the square root from the maximal intensity $\left(I_{0}\right)$ of the incident pulse: $A_{0}=\sqrt{I_{0}}$.

Dimensionless parameters for Eqs. (9) and (10) relate to the physical variables as follows:

$$
\begin{gathered}
\mathrm{z} \rightarrow \mathrm{z} / l_{\mathrm{dis}}, \quad t \rightarrow t / \tau_{p}, \quad l_{\mathrm{dis}}=\tau_{p}^{2} / D_{2}, \quad \theta=\left(2 \omega_{p}-\omega_{21}\right) T_{\perp}=\nu T_{\perp}, \\
\delta_{0}=\frac{4 \pi \omega_{p}^{2}}{c^{2}} \frac{\hbar}{k_{p}} r_{12}^{2} T_{\perp} N_{s} N_{0} \frac{1}{1+\theta^{2}} l_{\mathrm{dis}} A_{0}^{2}, \quad \xi=\delta_{0} \theta, \quad \tilde{\delta}=\hat{\varsigma} N_{0} 4 r_{12}^{2} T_{\perp} \frac{1}{1+\theta^{2}} \tau_{p} A_{0}^{4},
\end{gathered}
$$

where $\tau_{p}$ is the incident pulse duration. In accordance with introduced dimensionless variables, the value of parameter $D$ is equal to unity, $D=1$, so that we consider a medium with normal dispersion for the chosen wavelength of laser radiation. Therefore, SOD leads to a pulse spreading. Nevertheless, we also provide computer simulation for $D \neq 1$ to understand the role of dispersion affect.

Parameter $\delta_{0}$ characterizes laser energy depletion on the pulse dispersion length (the pulse after propagating a distance equal to the dispersion length is broaden by a factor $\sqrt{2}) .{ }^{54}$ Coefficient $\xi$ characterizes laser pulse self-action due to detuning of the doubled carrier frequency of wave packet from the frequency corresponding to the energy transition $2 \rightarrow 1$, if we consider a TPA, for example. The case of $\xi=0$ corresponds to optical pulse propagation in a medium with pure amplitude grating. It means an influence only of TPA (or SPA) on the 
laser pulse propagation. In the opposite case $(\xi \neq 0)$, the phase grating is also induced by the laser radiation. It should be mentioned that the positive sign of the parameter $\xi$ (this case is named by us as positive grating) corresponds to pulse compression and the laser pulse decompression occurs at the negative sign of this parameter (this case is named by us as negative grating).

Essentially, to observe the effect under consideration in physical experiments, it is necessary that the frequency detuning should be less than $0.1 \omega_{p}$, for example. If the laser radiation frequency is equal to $\omega_{p} \approx 2360 \mathrm{THz}(\lambda=800 \mathrm{~nm})$ and a time of transverse relaxation $T_{\perp}$ is about $10^{-13} \mathrm{~s},{ }^{47}$ then the dimensionless detuning parameter $\theta=\left(2 \omega_{p}-\omega_{21}\right) T_{\perp}=\xi / \delta_{0}$ should not exceed 20 dimensionless units. We stress a value of the parameter $\theta$ bellow.

Later in this paper, we provide an analytical consideration and numerical simulation for Eqs. (9) and (10) in dimensionless variables. We analyze a propagation of incident unchirped Gaussian pulse

$$
A(z=0, t)=A_{0}(t)=\exp \left\{-\left[\left(t-L_{t} / 2\right) / \tau\right]^{2}\right\}, \quad 0 \leq t \leq L_{t},
$$

where $\tau$ is a dimensionless pulse duration. We also follow the laser pulse center position

$$
\tau_{c}(z)=\int_{0}^{L_{t}}\left(t-L_{t} / 2\right)|A(z, t)|^{2} \mathrm{~d} t / \int_{0}^{L_{t}}|A(z, t)|^{2} \mathrm{~d} t .
$$

\section{Analytical Consideration}

\subsection{Nonlinear Geometric Optics Approximation for Incident Chirped Pulse Propagation}

Below, we develop the solution of Eqs. (9) and (10) in the framework of nonlinear geometric optics for a medium with SPA $(k=1)$, the function [Eq. (4)] for nanorod aspect ratio, and an incident chirped pulse. This analysis allows us to understand and explain the laser pulse propagation features. Let us note, this nonlinear geometric optics approach was widely used earlier with success for analysis of laser beams and pulses propagation in nonlinear media. As it is well-known, for this approach validity, it is necessary that the small-scale perturbations in the pulse shape and frequency distribution are absent. In this case, a pulse dispersion length is defined by the incident pulse duration and SOD influence appears qualitatively on this distance.

Let us represent the complex amplitude as $A=\sqrt{I} \exp (-i S), S$ is a real function describing a pulse phase and $I(z, t)=|A(z, t)|^{2}$ is a pulse intensity. We also introduce the instantaneous frequency $\Omega(z, t)=\frac{\partial S(z, t)}{\partial t}$ of the optical pulse and the temporal integral of the optical pulse intensity $P(z, t)=\int_{0}^{t} I(z, \tau) \mathrm{d} \tau$, passed through a medium section $z$ until time moment $t$ (this integral has unit as optical fluence). In nonlinear geometric optics approximation, Eqs. (9) and (10) are transformed to ${ }^{37}$

$$
\begin{aligned}
& \frac{\partial P}{\partial z}+2 D \Omega \frac{\partial P}{\partial t}-2 \frac{\delta_{0}}{\tilde{\delta}} f_{0}[\exp (-\tilde{\delta} P)-1]=0, \\
& \frac{\partial \Omega}{\partial z}+2 D \Omega \frac{\partial \Omega}{\partial t}+\xi \tilde{\delta} f_{0} \exp (-\tilde{\delta} P) I(z, t)=0,
\end{aligned}
$$

with the corresponding boundary conditions

$$
P(0, t)=P_{0}(t)=\int_{0}^{t} I(0, \tau) \mathrm{d} \tau, \quad \Omega(0, t)=\Omega_{0}(t)=\frac{\partial S(z=0, t)}{\partial t},
$$

and initial value for the nanorod aspect ratio $f_{0}=\varepsilon_{0}-1$. First terms in Eqs. (13)-(14) describe evolution of the temporal integral of the pulse intensity and the pulse instantaneous frequency along the $z$-coordinate, respectively. The second ones characterize their changing in time. And the last terms describe laser energy damping and frequency phase changing because of a nonlinear absorption and phase grating appearance, respectively. 
The solution of the Cauchy problem for Eqs. (13) and (14) can be developed, for example, by the characteristics method ${ }^{55}$ using

$$
\frac{\mathrm{d} z}{1}=\frac{\mathrm{d} t}{2 D \Omega}=\frac{\mathrm{d} P}{2 \frac{\delta_{0}}{\tilde{\delta}} f_{0}[\exp (-\tilde{\delta} P)-1]}=-\frac{\mathrm{d} \Omega}{\xi \tilde{\delta} f_{0} \exp (-\tilde{\delta} P) I(z, t)}
$$

The first equality [Eq. (16)] gives

$$
\frac{\mathrm{d} t}{\mathrm{~d} z}=2 D \Omega,
$$

which is the equation for characteristics. Remember that a characteristic is a curve in the plane $(z, t)$ along which the temporal integral of the pulse intensity $P(z, t)$ and instantaneous frequency $Q(z, t)$ remain constant or change according to ordinary differential equations with respect to the $z$-coordinate. Therefore, let us introduce a new variable for time (characteristic)

$$
\eta=t-2 D \int_{0}^{z} \Omega(t, x) \mathrm{d} x .
$$

Obviously, its value is equal to the time $t$ at the input section $z=0$ of a medium and remains constant along the characteristic. Therefore, each of the characteristics is defined by initial time moment $(t=\eta)$ in the input section $z=0$, and at a constant value $\eta$ we see "shifting" of this time corresponding to the $z$-section of a medium. In other words, each of the characteristics defines one-to-one correspondence between a time moment in the input section and a time moment in the current section $(z)$ of the medium.

If only a pure amplitude grating $(\xi=0)$ is induced by a laser radiation, the function $\Omega(z, \eta)$ is constant along the characteristics, $\Omega(z, \eta)=\Omega_{0}(\eta)$. Therefore, the relation between coordinates $\eta$ and $t, z$ follows from Eq. (17)

$$
\eta=t-2 D \Omega_{0}(\eta) z
$$

This case is investigated in Ref. 37, where a good quantitative agreement of a pulse center evolution, calculated using both the nonlinear geometric optics and computer simulation, was shown.

Along the characteristics, Eqs. (13) and (14) take the form

$$
\begin{gathered}
\frac{\mathrm{d} P(z, \eta)}{d z}-2 \frac{\delta_{0}}{\tilde{\delta}} f_{0}\{\exp [-\tilde{\delta} P(z, \eta)]-1\}=0, \\
\frac{\mathrm{d} \Omega(z, \eta)}{\mathrm{d} z}=-\xi \tilde{\delta} f_{0} \exp [-\tilde{\delta} P(z, \eta)] I(z, t) .
\end{gathered}
$$

Thus, evolution of the temporal integral of the pulse intensity can be written in an explicit form

$$
P(z, \eta)=\frac{1}{\tilde{\delta}} \ln \left[1+\exp \left(-2 \delta_{0} f_{0} z\right)\left\{\exp \left[\tilde{\delta} P_{0}(\eta)\right]-1\right\}\right] .
$$

From the above equation, the intensity distribution can be derived

$$
I(z, t)=\frac{\mathrm{d} P[z, \eta(z, t)]}{\mathrm{d} t}=\frac{I_{0}(\eta)}{1+\exp \left[-\tilde{\delta} P_{0}(\eta)\right]\left[\exp \left(2 \delta_{0} f_{0} z\right)-1\right]} \frac{\partial \eta}{\partial t} .
$$

If the characteristics can be approximated by the equation $\eta=t$, which means a weak delay in time caused by a propagation distance because of SOD $(D)$ influence or a pulse chirp $(\Omega)$ influence, it is possible to derive an explicit formula for the instantaneous frequency evolution by substituting Eqs. (22) and (23) for the pulse energy and intensity distribution into Eq. (21)

$$
\Omega(z, t)-\Omega_{0}(t)=\frac{\xi \tilde{\delta}}{2 \delta_{0}}\left[I(z, t)-I_{0}(t)\right], \quad I(z, t)=\frac{I_{0}(t)}{1+\exp \left[-\tilde{\delta} P_{0}(t)\right]\left[\exp \left(2 \delta_{0} f_{0} z\right)-1\right]} .
$$



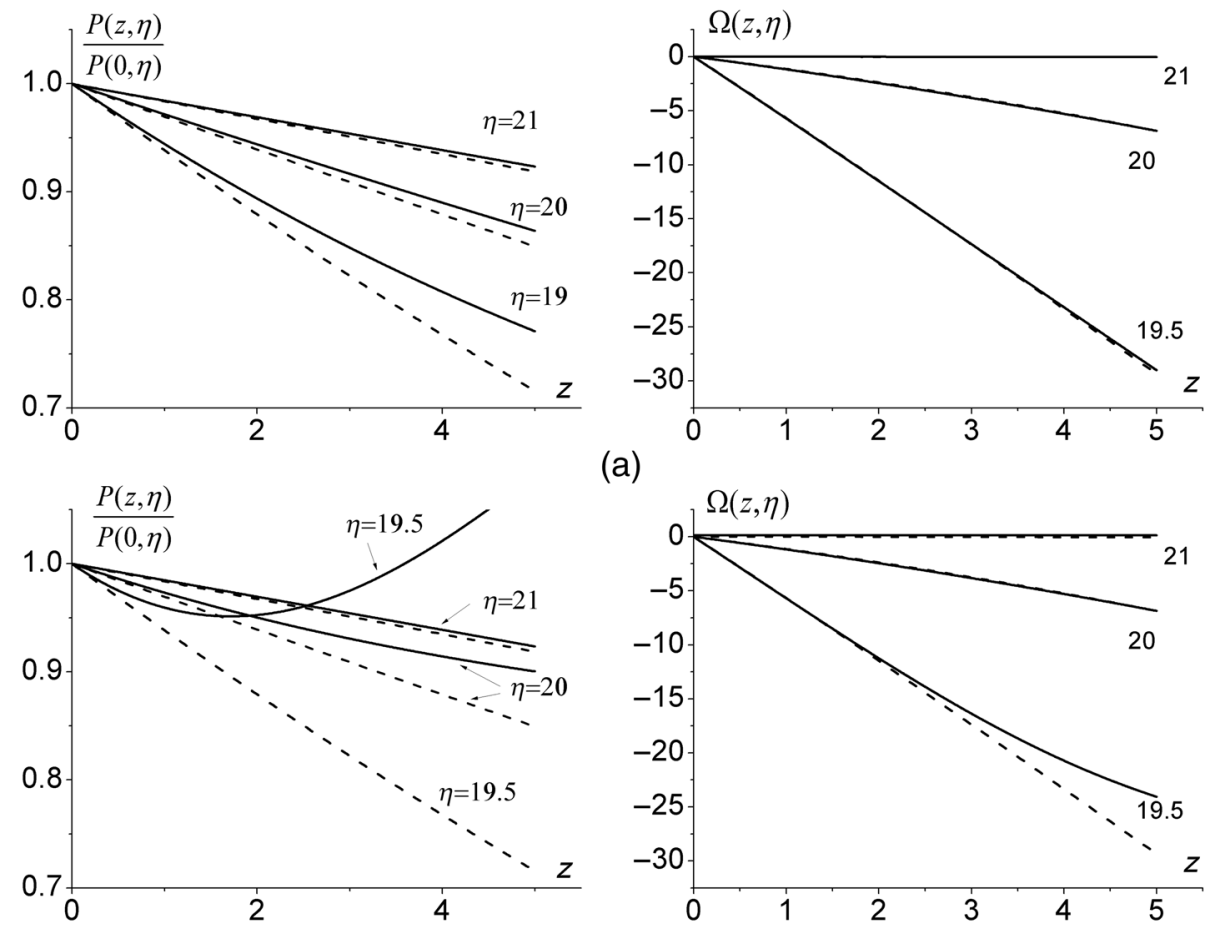

a)

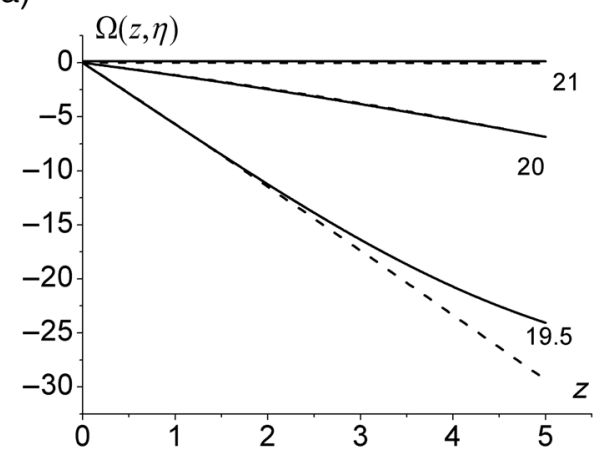

(b)
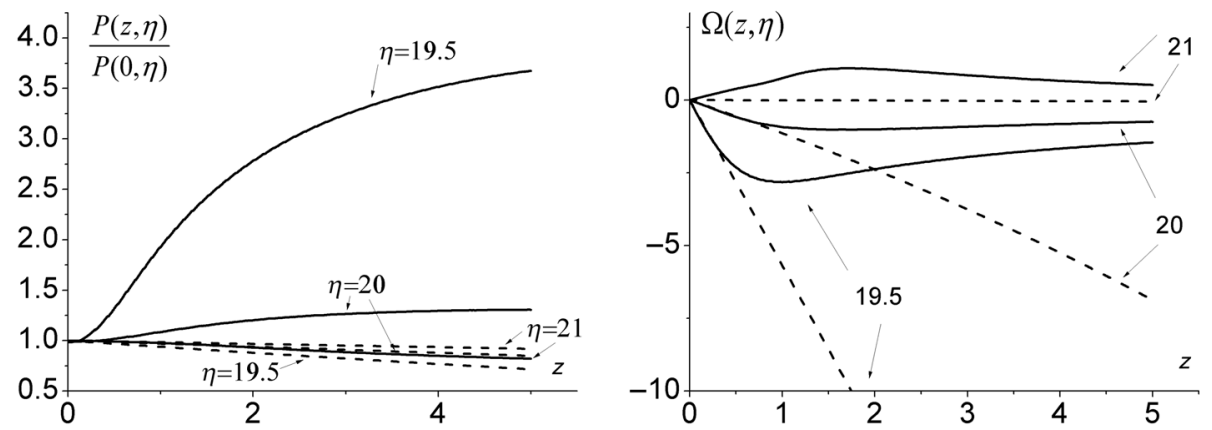

(c)

Fig. 3 Temporal integral of the optical pulse intensity $P(z, \eta)$ and instantaneous frequency $\Omega(z, \eta)$ evolution along the characteristics for the three values of $\eta$ (indicated by figures) at the incident Gaussian pulse propagation in a medium with SPA and (a) $D=10^{-4}$, (b) $10^{-3}$, (c) $0.1, \xi=5$, $\delta_{0}=0.05$, and $\tilde{\delta}=5$. Dashed lines correspond to the curves obtained using Eqs. (17), (20), and (23).

We see from Eq. (24) that due to the phase grating, the instantaneous frequency changes with time and a propagation distance if the pulse shape differs from its incident distribution.

To prove our analytical considerations, in Fig. 3, we compare the computer simulation results with the curves obtained using Eqs. (17), (22), and (24) for three different values of dimensionless $\operatorname{SOD}\left(D=10^{-4}, 10^{-3}, 0.1\right)$, and $\xi=5, \delta_{0}=0.05, \tilde{\delta}=5$. The dimensionless detuning parameter for these parameters is $\theta=100$, which corresponds to the frequency detuning about $0.5 \omega_{p}$. Maybe this value is big enough for practical application, but it allows us to demonstrate the pronounced validity of the solution obtained in the framework of nonlinear geometric optics. Solving Eq. (17) numerically together with Eqs. (9) and (10), we obtain the time $t_{z}(z, \eta)$ for three values of characteristic $\eta, \eta=(19.5,20,21)$, for example. [Remember that each characteristic is defined by time in the input section $(z=0, \eta=t)$, so that the value of $\eta$ defines a time in the section $z$ of a medium that corresponds to a time moment in the input section.) For each of the characteristics, we used Eq. (22) to calculate the laser pulse energy evolution $P(z, \eta)$ and Eq. (24) for the instantaneous frequency evolution under assumption $t=\eta$ (dashed lines in Fig. 3). This assumption implies that the characteristics 

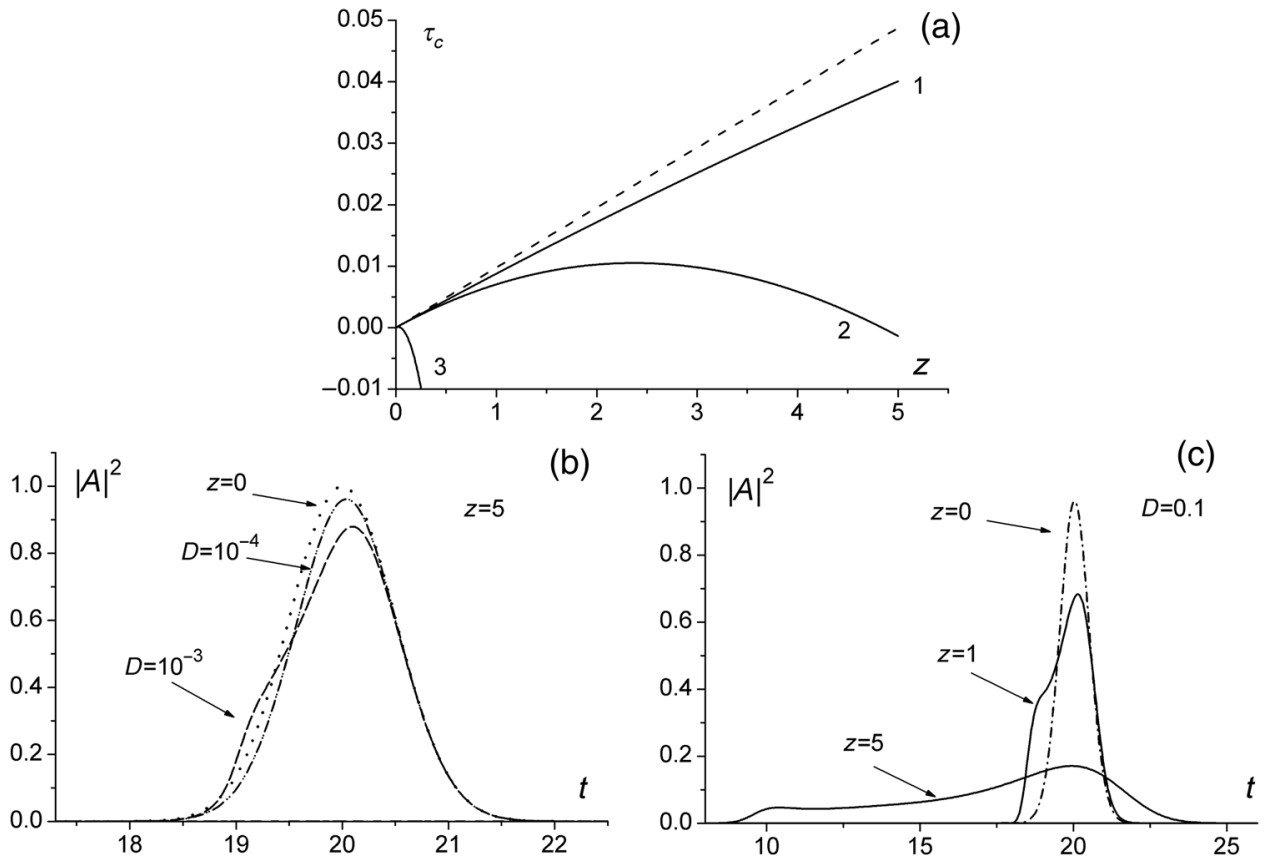

Fig. 4 (a) Pulse center shift evolution for the incident Gaussian pulse propagation in a medium with SPA and (1) $D=10^{-4}$, (2) $10^{-3}$, (3) 0.1 ; dashed line corresponds to the curve obtained using Eq. (21); (b) pulse shapes at $z=0$ (dotted line), 5 for $D=10^{-4}$ (dashed-dotted line), $10^{-3}$ (solid line); (c) pulse shapes at different sections for $D=0.1$; other parameters are $\xi=5, \delta_{0}=0.05$, $\tilde{\delta}=5$.

are straight lines in the plane $(z, t)$. This approach is confirmed by computer simulation if the SOD is quite small or we analyze the trailing edge of the pulse where the front of nanorods reshaping is absent. Indeed, we see a validation of this statement for $D=10^{-4}, 10^{-3}$ or $\eta=20, D=0.1$ at the whole propagation distance, or at the distance $z<0.5$ if $\eta=19.5$, 21 for $D=0.1$. It is important to stress that the characteristics are close to the straight line for the other part of the propagation distance. The laser pulse energy and instantaneous frequency evolution calculated using Schrödinger equation are depicted as solid lines in Fig. 3.

From Fig. 3, the nonlinear geometric optics approximation is valid along the total propagation distance if SOD is small $\left[D=10^{-4}, 10^{-3}\right.$, Figs. 3(a) and 3(b)] which is valid for the pulse with duration of a few picoseconds or greater. In these cases, Eq. (24) describes the instantaneous frequency evolution quite well [compare the solid and dashed lines in Figs. 3(a) and 3(b)]. If a pulse dispersion is large enough $(D=0.1$, that corresponds to the pulse duration of about $0.5 \mathrm{ps}$ in our notations), the nonlinear geometric optics approximation is valid for propagation distances less than 1 dimensionless unit [Fig. 3(c)], which corresponds to one-tenth of the dispersion length. We see that the biggest discrepancy between the curves is observed for characteristic $\eta=19.5$. At this time moment $t=19.5$, the nanorods reshaping front takes place and, therefore, a high absorption of laser energy occurs. As a result, the pulse front velocity gradually decreases and the pulse front undergoes distortions, including the instantaneous frequency while the pulse back remains unchanged for the larger propagation distance. As a result, a nonlinear geometric optics approximation is valid near the pulse back at a long propagation distance.

In Fig. 4, the pulse center shift evolution, calculated using Schrödinger equation (solid lines) and obtained on the basis of Eqs. (12) and (23) under assumption $t=\eta$ (dashed line), is depicted for the considered parameters and $D=10^{-4}, 10^{-3}$. The best coincidence between the solid and dashed curves is observed for $D=10^{-4}$. For $D=10^{-3}$, a self-trapping of laser radiation by the nanorods reshaping front begins to play a dominant role. As a consequence, a discrepancy between the curves is noticeable for $z>0.5$. If $D=0.1$, there is also a significant difference between the calculated on the base of computer simulation curve and the analytical curve due to the self-trapping effect. Obviously, it cannot be described without taking into account the SOD. In this case, the curves coincide only at the propagation distance less than 0.1 dimensionless 
units as we mentioned earlier. To illustrate self-trapping of laser radiation by the nanorods reshaping front, we show in Figs. 4(b) and 4(c) the pulse shapes at different section of the medium. While for $D=10^{-4}$, the pulse shape at section $z=5$ is similar to the incident pulse shape, for $D=10^{-3}$, the pulse front is self-trapped [Fig. 4(b)]. Self-trapping of the pulse by the nanorods reshaping front is well seen for $D=0.1$ even at section $z=1$ [Fig. 4(c)].

\subsection{Soliton-Like Solution}

In this section, we develop a soliton-like solution for the pulse propagation in a medium with TPA and nanorods for $f(\varepsilon)$ in Eq. (4). Computer simulation ${ }^{37}$ has earlier demonstrated a soliton formation. Therefore, we develop below an approximate analytical solution that clarifies the main features of soliton appearance (detailed derivation of equations for this solution is presented in the Appendix). For this aim, let us represent the complex amplitude in a soliton-like form

$$
\begin{gathered}
A(z, t)=B(z) c h^{-1}(\zeta) \exp [-i s(z, \zeta)], \quad f(z, t)=f(z, \zeta), \quad \zeta=\left[t-t_{c}(z)\right] / \tau_{s}(z), \\
B(0)=B_{0}, \quad t_{c}(0)=t_{0}, \quad \tau_{s}(0)=\tau_{s 0},
\end{gathered}
$$

and the pulse phase $s(t, \zeta)$ as

$$
s(z, \zeta)=a(z)+b(z) \zeta+h(z) \ln [\operatorname{ch}(\zeta)], \quad a(0)=a_{0}, \quad b(0)=b_{0}, \quad h(0)=h_{0} .
$$

Functions $B(z), s(z, \zeta), t_{c}(z)$, and $\tau_{s}(z)$ describe the soliton amplitude, phase distribution, pulse center, and its duration, respectively, with their initial values $B_{0}, t_{0}$, and $\tau_{s 0}$ for time moment $t=0$. It should be kept in mind that the soliton appears after the laser pulse propagates along a certain distance. That is why the initial values of introduced parameters do not coincide with the incident pulse position.

Parameters $a_{0}, b_{0}$, and $h_{0}$ define the incident pulse phase distribution. Function $a(z)$ describes a soliton phase shift along the $z$-coordinate and is unchangeable in time. Functions $b(z)$ and $h(z)$ describe the soliton frequency evolution along the propagation coordinate and a pulse chirp evolution, respectively. As mentioned earlier, we derived self-similar solutions for laser pulse propagation in the media with nonlinear MPA. ${ }^{39,40}$ Their pulse phase distributions are described by the third term in Eq. (27) with the constant coefficient at this term $(h(z)=$ const along the $z$-coordinate). In this paper, we consider the similar term taking into account its dependence on the $z$-coordinate. Moreover, we also consider the linear dependence of the phase on the coordinate $\zeta$ [the second term in Eq. (27)] to describe the phase asymmetric dependence on time due to the absorption of laser radiation energy by nanorods. We suppose that the time-independent first term in Eq. (27), as well as the coefficient $b(z)$, depends on the $z$-coordinate. Note that the pulse phase distribution under consideration corresponds to the "classical" soliton phase distribution if the pulse chirp is absent $h(z)=0$.

The following equation can be obtained by integrating Eq. (10) after substituting Eqs. (4) and (25) into Eq. (10):

$$
f(z, \zeta)=f_{0} \exp \left[-\tilde{\delta} B^{4} \tau_{s}\left(t h \zeta-1 / 3 t h^{3} \zeta+2 / 3\right)\right] .
$$

Dependence [Eq. (28)] describes a nanorods reshaping front under the action of laser radiation, and the corresponding aspect ratio evolution in time is depicted in Fig. 5. We see a pronounced reshaping front of the nanorods, and its evolution in time is in a good agreement with computer simulation results (see Sec. 4).

Substituting Eqs. (25), (27), (28) into Eq. (9), we get after certain transformations (see the Appendix) the following set of equations:

$$
\begin{gathered}
\tau_{s}(z)=\tau_{s 0} \\
3 \frac{\mathrm{d} \ln B}{\mathrm{~d} z}+\delta_{0} f_{0} B^{2} \exp \left(-2 / 3 \tilde{\delta} B^{4} \tau_{s 0}\right)\left[1 / 2\left(\tilde{\delta} B^{4} \tau_{s 0}\right)^{2}+2\right]=0
\end{gathered}
$$




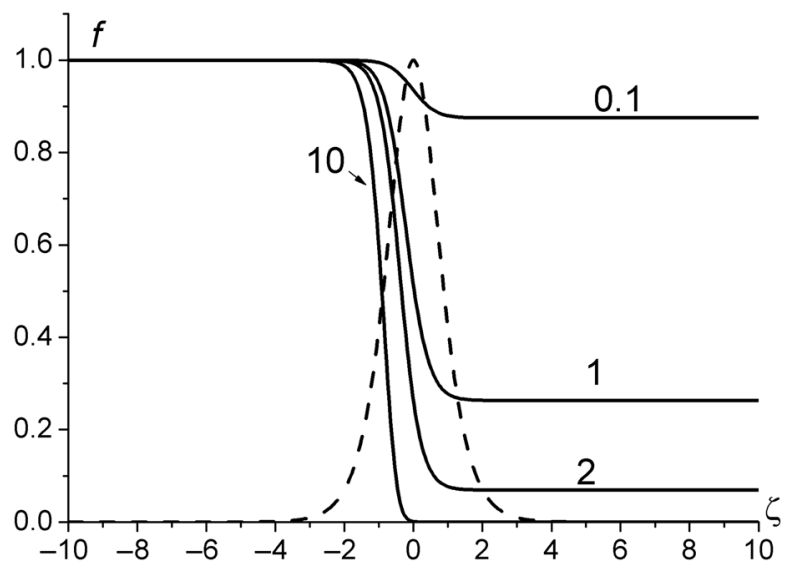

Fig. 5 Aspect ratio evolution (dependence Eq. (28), solid lines) for various values of $\tilde{\delta} B^{4} \tau_{s}$ (denoted by figures). Dashed line shows the soliton shape described by Eq. (25).

$$
\begin{gathered}
\frac{\mathrm{d} t_{c}}{\mathrm{~d} z}-2 \frac{D}{\tau_{s}} b-\delta_{0} f_{0} \tilde{\delta} \tau_{s}^{2} B^{6} \exp \left(-2 / 3 \tilde{\delta} B^{4} \tau_{s}\right)=0 \\
\frac{\mathrm{d} a}{\mathrm{~d} z}-b \frac{1}{\tau_{s}} \frac{\mathrm{d} t_{c}}{\mathrm{~d} z}+\frac{D}{\tau_{s}^{2}}\left(1+b^{2}\right)-\xi f_{0} B^{2} \exp \left(-2 / 3 \tilde{\delta} B^{4} \tau_{s}\right)=0 \\
\frac{\mathrm{d} b}{\mathrm{~d} z}-h \cdot \frac{1}{\tau_{s}} \frac{\mathrm{d} t_{c}}{\mathrm{~d} z}+2 \frac{D}{\tau_{s}^{2}} \cdot b \cdot h+\xi f_{0} \tilde{\delta} \tau_{s} B^{6} \exp \left(-2 / 3 \tilde{\delta} B^{4} \tau_{s}\right)=0 \\
-3 D h+\delta_{0} f_{0} \tau_{s 0}^{2} B^{2} \exp \left(-2 / 3 \tilde{\delta} B^{4} \tau_{s 0}\right)\left[1 / 2\left(\tilde{\delta} B^{4} \tau_{s 0}\right)^{2}-1\right]=0 \\
D\left(h^{2}-2\right)+\xi f_{0} \tau_{s}^{2} B^{2} \exp \left(-2 / 3 \tilde{\delta} B^{4} \tau_{s}\right)\left[1-1 / 2\left(\tilde{\delta} B^{4} \tau_{s}\right)^{2}\right]=0 .
\end{gathered}
$$

The following consequence of Eqs. (34) and (35) is more convenient for analysis

$$
\delta_{0} h^{2}-3 \xi h-2 \delta_{0}=0 .
$$

In particular, from the above equation, we can get

$$
h=\frac{3 \xi / \delta_{0} \pm \sqrt{9\left(\xi / \delta_{0}\right)^{2}+8}}{2}=\frac{3 \theta \pm \sqrt{9 \theta^{2}+8}}{2},
$$

for a medium with absorption. Expression [Eq. (37)] means existence of two frequency chirps with different signs and the constant value of function $h(z)$. The positive sign corresponds to the slowing down soliton while the negative sign corresponds to the accelerating soliton. On the other hand, Eq. (34) defines a relation between the soliton duration and its intensity $\left(B^{2}\right)$ and the pulse chirp $(h)$. These two equations should not contradict each other. We see from Eq. (34) that the product $D h$ takes a certain sign depending on the amplitude and duration of the soliton. So, in a medium with normal dispersion, the amplitude and duration of the soliton satisfy the inequality $B^{4} \tau_{s 0}>\sqrt{2 / \tilde{\delta}}$ for a positive value of $h$, and the opposite inequality takes place for its negative value. Obviously, in dependence of this inequality, one of the signs for chirp can lead to a physically impracticable sign of the soliton intensity. Therefore, if the pulse duration, intensity and absorption parameter $\tilde{\delta}$ are specified, only one type of the solitons occurs.

To write the soliton amplitude $B(z)$ in the implicit form, we get from Eqs. (30) and (34) the following equation:

$$
\frac{\mathrm{d} \ln B}{\mathrm{~d} z}+\frac{D h}{\tau_{s 0}^{2}} \frac{\left(\tilde{\delta} B^{4} \tau_{s 0}\right)^{2}+4}{\left(\tilde{\delta} B^{4} \tau_{s 0}\right)^{2}-2}=0
$$

which can be integrated 


$$
\frac{\left[\left(\tilde{\delta} B^{4} \tau_{s 0}\right)^{2}+4\right]^{3}}{\left(\tilde{\delta} B^{4} \tau_{s 0}\right)^{2}}=\frac{\left[\left(\tilde{\delta} B_{0}^{4} \tau_{s 0}\right)^{2}+4\right]^{3}}{\left(\tilde{\delta} B_{0}^{4} \tau_{s 0}\right)^{2}} \exp \left(-16 \frac{D h}{\tau_{s 0}^{2}} z\right)
$$

Therefore, Eq. (39) together with Eqs. (34) and (37) allows us to obtain the amplitude $B$, the pulse chirp, and the pulse duration at each section of the medium.

Taking into account Eqs. (31) and (33), one can write the equation for the pulse frequency shift evolution

$$
\frac{\mathrm{d} b}{\mathrm{~d} z}=\left(h \cdot \delta_{0}-\xi\right) f_{0} \tilde{\delta} \tau_{s 0} B^{6} \exp \left(-2 / 3 \tilde{\delta} B^{4} \tau_{s 0}\right)
$$

Since the pulse chirp and the pulse amplitude are defined from the above equations, we can solve this equation and obtain the soliton center $t_{c}(z)$ evolution [see Eq. (31)] as well as timeindependent part of the soliton phase $a(z)$ evolution [Eq. (32)].

One more important conclusion can be made at the analyzing the following equality, $\left(h \cdot \delta_{0}-\xi\right)=\delta_{0}(h-\theta)=\delta_{0}\left(\theta \pm \sqrt{9 \theta^{2}+8}\right) / 2$, which is valid for the coefficient at the right hand of Eq. (40). Taking into account that the sign of this coefficient coincides with the sign of the chirp $h$, which is given by Eq. (37), we conclude that the pulse frequency shift $b(z)$ increases for a positive chirp and it decreases for a negative chirp regardless of which of the phase gratings - positive or negative-is induced by laser radiation.

To verify the analytical solution, we discuss in Sec. 4 the computer simulation results and show that a soliton formation takes place for a wide range of dimensionless absorption amplitude $\delta_{0}$, from 0.005 to 0.1 . Taking this into account, for qualitative analysis providing and further simplifying of expressions with respect to soliton parameters, we assume negligible absorption $\left(\delta_{0} \cong 0\right)$. It allows to illustrate the influence of certain physical factors on a laser pulse propagation. It should be stressed that in the framework of this analysis the pulse chirp is absent because it appears due to nonlinear absorption as was mentioned earlier. In this case, Eqs. (30), (31), (34), and (35) transform to simple equations

$$
\frac{\mathrm{d} \ln B}{\mathrm{~d} z}=0, \quad h=0, \quad \frac{1}{\tau_{s 0}} \frac{\mathrm{d} t_{c}}{\mathrm{~d} z}-2 \frac{D}{\tau_{s 0}^{2}} b=0, \quad B^{2} \exp \left(-2 / 3 \tilde{\delta} B^{4} \tau_{s 0}\right)\left[1-1 / 2\left(\tilde{\delta} B^{4} \tau_{s 0}\right)^{2}\right]=\frac{2 D}{\xi f_{0} \tau_{s 0}^{2}},
$$

which have the following solution:

$$
B(z)=B_{0}, \quad h(z)=0, \quad t_{c}(z)=2 \frac{D}{\tau_{s 0}} \int_{0}^{z} b(\eta) \mathrm{d} \eta+t_{0} .
$$

Since the soliton amplitude $B(z)=B_{0}$ and duration $\tau_{s}(z)=\tau_{s 0}$ are constant, we get from Eqs. (32), (40), and (42) the linear law of the frequency shift changing $b(z)$

$$
b(z)=b_{0}-\xi f_{0} \tilde{\delta} \tau_{s 0} B_{0}^{6} \exp \left(-2 / 3 \tilde{\delta} B_{0}^{4} \tau_{s 0}\right) z,
$$

and quadratic dependence of the soliton center $t_{c}(z)$ evolution

$$
t_{c}(z)=t_{0}+2 \frac{D}{\tau_{s 0}} b_{0} z-\frac{D}{\tau_{s 0}} \xi \tilde{\delta} f_{0} B_{0}^{6} \tau_{s 0} \exp \left(-2 / 3 \tilde{\delta} B_{0}^{4} \tau_{s 0}\right) z^{2}
$$

and cubic dependence of the soliton homogeneous phase shift $a(z)$

$$
\begin{aligned}
a(z)= & a_{0}+\left[\frac{D}{\tau_{s 0}^{2}}\left(b_{0}^{2}-1\right)+\xi f_{0} B_{0}^{2} \exp \left(-2 / 3 \tilde{\delta} B_{0}^{4} \tau_{s 0}\right)\right] z \\
& -\frac{D}{\tau_{s 0}} b_{0} \xi f_{0} \tilde{\delta} B_{0}^{6} \exp \left(-2 / 3 \tilde{\delta} B_{0}^{4} \tau_{s 0}\right) z^{2}+\frac{1}{3} D\left[\xi f_{0} \tilde{\delta} B_{0}^{6} \exp \left(-2 / 3 \tilde{\delta} B_{0}^{4} \tau_{s 0}\right)\right]^{2} z^{3} .
\end{aligned}
$$

Equations (43)-(45) give the main features of soliton-like solution in the framework of used approach, namely, the constant value of the amplitude $B(z)=B_{0}$, linear dependence of the pulse instantaneous frequency $-\frac{\partial s}{\partial t}=-\frac{b(z)}{\tau_{s 0}}$ on the $z$-coordinate, and quadratic dependence of 
Trofimov and Lysak: Superluminality phenomenon at a femtosecond laser pulse propagation...

the soliton center shift $t_{c}(z)$ on the $z$-coordinate. Equation (43) shows that the pulse frequency shift $b(z)$ decreases along the $z$-coordinate for a positive phase grating $(\xi>0)$ and it increases for a negative phase grating $(\xi<0)$. The similar dependence takes place for the soliton center $t_{c}(z)$ evolution in a medium with normal dispersion $(D>0)$. For example, if $b_{0}=0$, the soliton center shifts into the area of time decreasing (accelerating of pulse) for a positive phase grating and into the area of time increasing (slowing down of pulse) for a negative phase grating. The opposite situation occurs in a medium with anomalous dispersion $(D<0)$.

These features of soliton-like propagation obtained in a very simple way are confirmed by the computer simulation results discussing in Sec. 4. Of course, the pulse chirp introduces more complicated features in laser pulse propagation. Let us stress that the "classical" soliton possesses unchangeable instantaneous frequency.

\section{Computer Simulation Results}

Below, we discuss computer simulation results for the propagation of an incident Gaussian pulse $(\tau=1)$ at $D=0.1$ in a medium with induced positive phase-amplitude grating $(\xi=5,2.5$, 1.25 ) for dimensionless nanorods reshaping strength $\tilde{\delta}=5$ and various values $\left(\delta_{0}=0.005\right.$, $0.05,0.1)$ of the laser energy depletion due to TPA. In Sec. 4.1, we present the results for the dependence $f(\varepsilon)$ in Eq. (4) with the initial value of nanorod aspect ratio $\varepsilon_{0}=2$, and in Sec. 4.2, we discuss the computer simulation results obtained for the dependence $f(\varepsilon)$ in Eq. (5) with $\varepsilon_{0}=2.6$.

It should be noted that the ratio between the dimensionless absorption parameter and the strength of phase grating $\theta=\xi / \delta_{0}$ (which is the dimensionless frequency detuning) for the given set of dimensionless parameters takes the values from $10^{3}$ to 12.5 . As was mentioned in Sec. 2, the value of $\theta$ equal 20 corresponds to $0.1 \omega_{p}$ if the laser radiation frequency is equal to $\omega_{p} \approx 2360 \mathrm{THz}(\lambda=800 \mathrm{~nm})$. So, the considering values of $\theta$ from 50 to 12.5 correspond to the frequency detuning from $\approx 0.2 \omega_{p}$ to $\approx 0.05 \omega_{p}$. Nevertheless, we also use large value of $\theta$ to confirm our analytical considerations.

Let us notice that a laser pulse interaction with nanorods near the nonlinear absorption resonance, which is described by function $f(\varepsilon)$ in Eq. (5) instead of Eq. (4), does not influence on the dimensionless frequency detuning $\theta$, but this function type strongly changes the optical energy absorption and the strength of induced phase grating, and, thus, decrease the propagation distance at which the effects under consideration can be observed.

\subsection{Soliton Formation Far from Nonlinear Response Resonance}

As is well seen from Fig. 2, the function $f(\varepsilon)$ in Eq. (4) approximates quite well the dependence [Eqs. (6) and (7)] for aspect ratio at satisfying the inequality $\varepsilon \leq \varepsilon_{0}=2$. At laser pulse propagation in such medium, the incident pulse splits into two subpulses for a wide range of parameters. As a rule, a soliton formation and light acceleration take place. In Sec. 4.1.1, the details of this effect are discussed for small depletion of the laser energy $\left(\delta_{0}=0.005\right)$ and $\xi=5$. For this case, the frequency detuning $\left(\theta=10^{3}\right)$ is very big for practice at application. Nevertheless, we consider this case to prove our analytical results developed in Sec. 3.2. In Sec. 4.1.2, we compare the computer simulation results for various values of a medium absorption and other parameters of the pulse propagation. Certain sets of them correspond to their achievement in physical experiments.

\subsubsection{Soliton formation under small depletion of laser energy}

The features of femtosecond pulse propagation in the medium with $f(\varepsilon)$ in Eq. (4), and $D=0.1$ and positive amplitude grating $(\xi>0)$, induced by laser radiation, and small depletion of laser energy as well as the physical mechanism for this phenomenon occurrence were discussed in Ref. 37. Nevertheless, for further consideration, it is important to give briefly these details below.

At the initial stage of propagation, the laser pulse is splitting into two subpulses (Fig. 6). The left one transforms into a soliton and propagates with high velocity without changing 

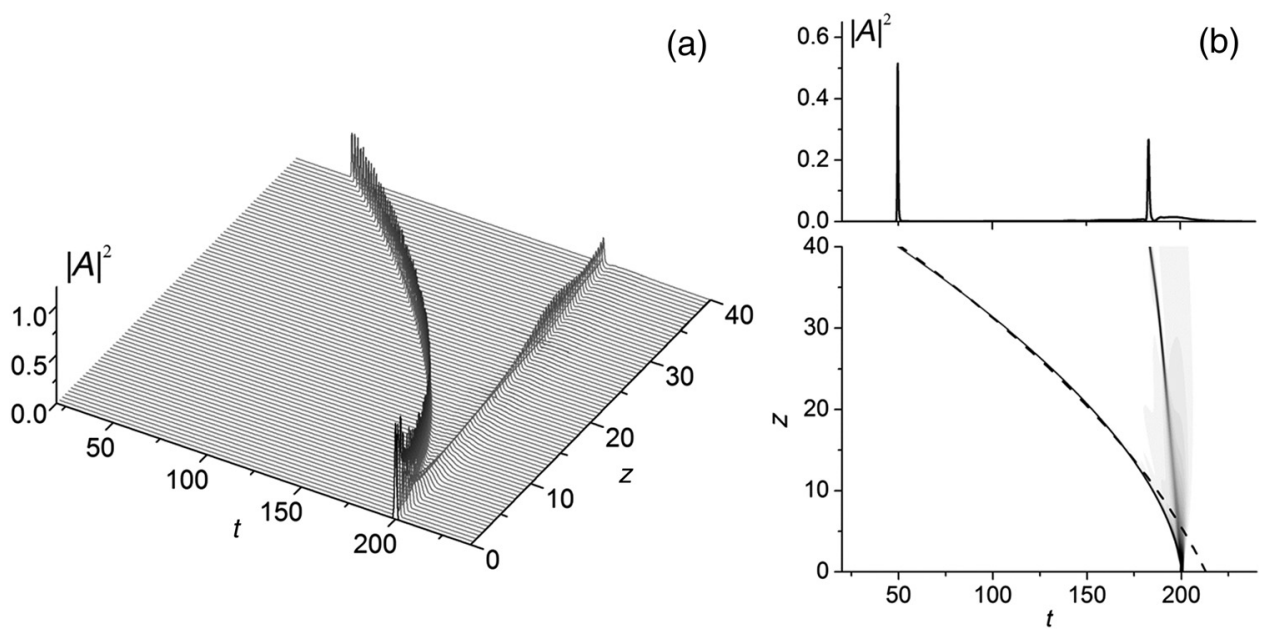

Fig. 6 (a) Soliton formation and (b) maximal intensity position for both subpulses at the incident Gaussian pulse propagation for the positive phase amplitude grating $\xi=5$ and $\delta_{0}=0.005, \tilde{\delta}=5$. Dashed line in (b) is given by Eq. (47). The top plane in (b) shows subpulse shapes at section $z=40$.

its shape for at least 30 dimensionless units [Fig. 6(b)]. The right subpulse also gradually transforms into a soliton. However, its velocity corresponds to the dispersion spreading of the pulse in a linear medium while the left subpulse velocity exceeds it many times. Due to TPA, the maximal intensity of both subpulses decreases very slowly at a long propagation distance [Fig. 7(b)]. This is the consequence of the influence of the nonlinear absorption and a pulse chirp appearance due to a phase grating and an amplitude grating.

The physical mechanism for light acceleration and soliton formation is as follows. ${ }^{37}$ A positive phase grating, which is formed at the pulse propagation, leads to a negative chirp of the pulse. On the other hand, a positive chirp of the pulse also appears due to the normal dispersion. As a result, waves with lower frequencies go to the pulse trailing edge and waves with higher frequencies go to its leading edge. This leads to the pulse compression, the wave packet power density grows, and the nanorods reshaping increases due to the high intensity waves absorption. The waves with lower frequencies are concentrated in the left subpulse while the waves with higher frequencies are concentrated in the right subpulse. So, the pulse splitting into two subpulses occurs, and the left subpulse moves with higher velocity than the right subpulse does if an optical radiation propagates in a medium with normal dispersion. The further compression of the subpulses due to the action of positive phase-amplitude grating is restricted by the normal dispersion action and laser energy absorption. Essentially, after a certain propagation distance, each of the subpulses is transformed into a soliton and becomes trapped at the boundary between domains with high and low nanorod aspect ratio.
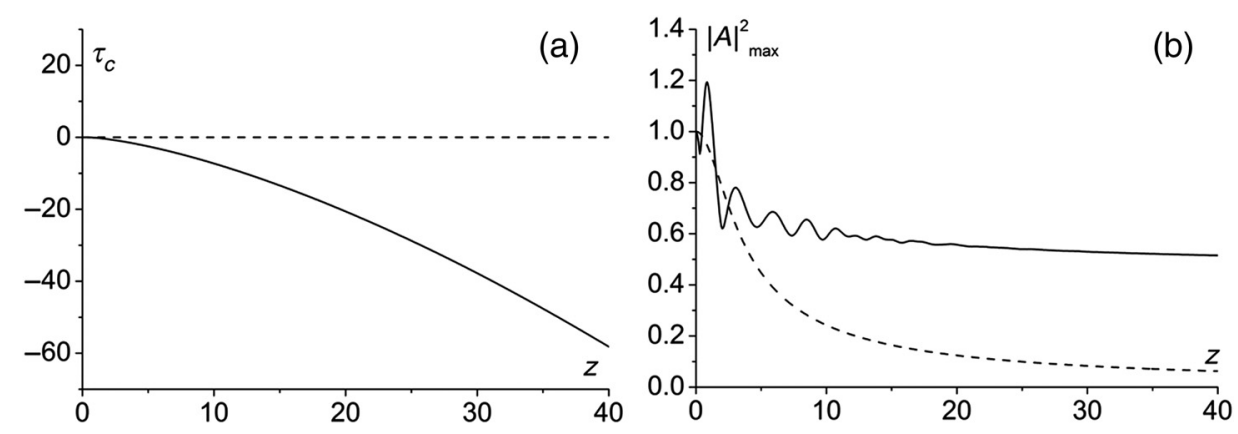

Fig. 7 (a) Pulse center shift along the z-coordinate, calculated using Eq. (12) and (b) maximal intensity evolution in a medium with the positive phase amplitude grating $\xi=5, \delta_{0}=0.005$, and $\tilde{\delta}=5$. Dashed lines correspond to the pulse propagation in a linear medium. 
Trofimov and Lysak: Superluminality phenomenon at a femtosecond laser pulse propagation...

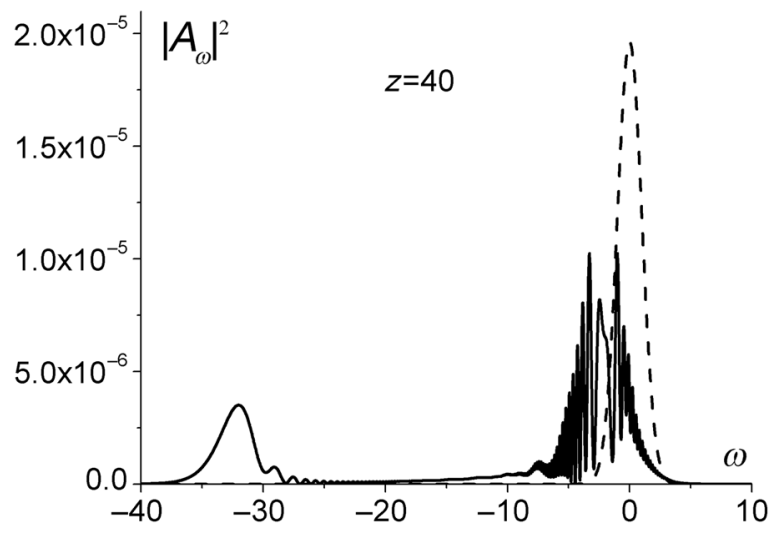

Fig. 8 Spectrum of the pulse, propagating in a medium with the positive phase grating $(\xi=5)$, $\delta_{0}=0.005, \tilde{\delta}=5$. Dashed line corresponds to the incident pulse spectrum.

As for a pulse spectrum evolution, we see that the influence of the positive phase grating results in a permanent shift of higher frequency waves to the subpulse front, where they are absorbed by nanorods. So, the spectrum of both subpulses is continuously shifting into the range of lower frequencies, especially for the high velocity left subpulse (Fig. 8). Therefore, the velocities of the subpulses grow. Nevertheless, the right subpulse velocity is close to the velocity of the pulse propagating in a linear medium. This is a consequence of low nanorod aspect ratio for the right subpulse. As a result, its self-action decreases and the subpulse compression also decreases. However, the right subpulse trapping occurs at a lower peak intensity in comparison with the left subpulse intensity. Moreover, this subpulse consists of higher frequency waves in comparison with the left subpulse. That is why its velocity is much smaller than the left subpulse velocity, though it is bigger than the velocity of the pulse, propagating in a linear medium. Due to both subpulses accelerating, the center of the whole laser pulse is shifting permanently in the direction of time decreasing [fast light, Fig. 7(a)].

It should be stressed that the left soliton shape can be approximated quite well by the function $^{37}$

$$
I_{0} c h^{-2}\left[\left(t-t_{c}\right) / \tau_{a p}\right]
$$

with $\tau_{a p}=0.3, I_{0}=0.56$, and $t_{c}=152.57$. We use these approximation parameters to confirm the results of our analytical consideration (Sec. 3.2). Let us note that Eq. (35) is valid for $\tau_{s 0}=0.345$ if the pulse intensity is chosen as $I_{0}=B_{0}^{2}=0.56$ and $h=0$ (which is valid for negligible absorption). We see that this pulse duration is in a good agreement with the value $\tau_{a p}=0.3$. The maximal intensity position $t_{c}(z)$ for the left subpulse can be approximated by the following quadratic function [shown by the dashed line in Fig. 6(b)]:

$$
t_{c}(z)=215-3 z-0.05 z^{2}
$$

that is similar to the corresponding Eq. (44). However, the coefficient at the term $z^{2}$ in Eq. (44), calculated for the parameters of the approximation [Eq. (46)], is equal to -0.3 , which is six times bigger than the corresponding coefficient at the same term in Eq. (47). Obviously, this difference is a consequence of the soliton appearance after the pulse passed the certain distance along which its shape is not a soliton-like shape. In our point of view, it is essential that the quadratic dependence of $t_{c}(z)$ obtained in Sec. 3.2 for approximate analytical solution, is confirmed by computer simulation result.

\subsubsection{Influence of the pulse energy depletion and the strength of phase grating on soliton formation}

In this section, we demonstrate that the incident pulse splitting into two subpulses, and soliton formation, and light acceleration, described in Sec. 4.1.1, takes place for a wide range of the 

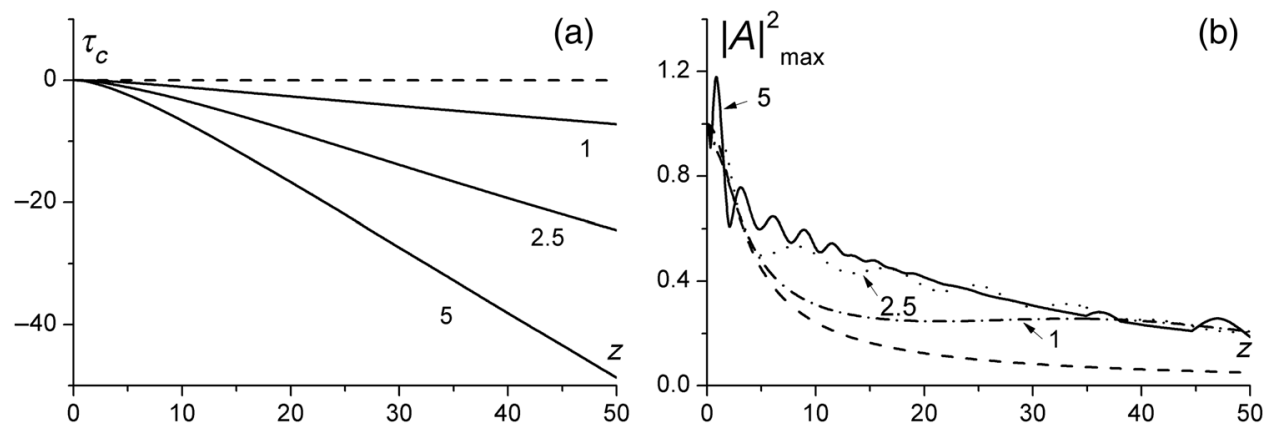

Fig. 9 (a) Pulse center shifting along the z-coordinate, calculated using Eq. (12) and (b) maximal intensity evolution for the positive phase amplitude grating (parameter $\xi$ values are denoted by figures) and parameters: $\delta_{0}=0.05, \tilde{\delta}=5$. Dashed lines correspond to the pulse propagation in a linear medium.

problem parameters with $f(\varepsilon)$ in Eq. (4), and $D=0.1$, and positive phase grating, induced by laser radiation. It is important to stress that we also consider a set of parameters that allows to observe these effects in practice.

The pulse center shifting along the $z$-coordinate and maximum intensity evolution for the pulse energy depletion, which is 10 times greater than that discussed in Sec. 4.1.1, $\delta_{0}=0.05$, are shown in Fig. 9 for three values of phase grating strength, $\xi=5,2.5$, 1 . One of these parameters corresponds to quite realistic values of frequency detuning, $\theta=100,50,20$. For all values of frequency detuning, the pulse center shifting into the area of time decreasing is well seen. The shift is the greatest for the strongest phase grating $(\xi=5)$. Nevertheless, it is less than the pulse shifting observing for $\delta_{0}=0.005$ [compare Fig. 7(a), solid line, and Fig. 9(a), line 5]. Ten times increasing of laser energy depletion results in three times the maximal intensity decreasing [compare Fig. 7(b), solid line, and Fig. 9(b), lines 1, 2.5, and 5]. For the biggest strength of phase grating $(\xi=5)$, the maximal intensity growth occurs at the initial stage of the pulse propagation as a result of its compression due to the induced nonlinear chirp. Further evolution of the pulse maximal intensity is characterized by the intensity oscillations. These oscillations are the result of the competing processes of nonlinear absorption and compression due to the chirp appearance for the pulse. Indeed, with the pulse intensity growth, the absorption of laser radiation also grows. This results in the pulse intensity decreasing. Then, the pulse compression due to the induced nonlinear chirp causes the pulse intensity growth once again and so on. Each of the next intensity peaks is less than the previous because of the laser radiation absorption. The intensity oscillations are also well seen for $\xi=2.5$.

Let us note that the pulse maximal intensity decreasing depends mainly on the medium absorption, whereas the pulse center shifting depends mainly on the phase grating strength. Obviously, the stronger the strength of the phase grating, the stronger the induced chirp of the pulse, and the greater is the left subpulse velocity.

The incident pulse splitting into two subpulses is depicted in Fig. 10. For the strong phase grating $(\xi=5)$, up to the section $z=50$, both subpulses are transformed into solitons with equal maximal intensities [Fig. 10(a)]. For the less strength of phase grating [ $\xi=2.5$, Fig. 10(b)], only the left subpulse has a soliton-like form at $z=50$, while for $\xi=1$ the left subpulse is not yet separated at all from the right subpulse [minimum value of the pulse intensity between the subpulses is about 0.003, which is a quite low value, Fig. 10(c)]. The full separation of the pulses takes place for a much more propagation distance. Nevertheless, its shape is close to a soliton one, and its shift in the area of time decreasing is about $7.5 \mathrm{ps}$ for a 500 -fs incident pulse [Fig. 10(c), incident pulse is shown by a dashed line].

\subsection{Soliton Formation for Strong Nonlinear Dependence of Laser Energy Absorption}

In this section, we discuss the features of soliton formation and light acceleration at the strong nonlinear dependence of laser energy absorption on the aspect ratio, which occurs near its 

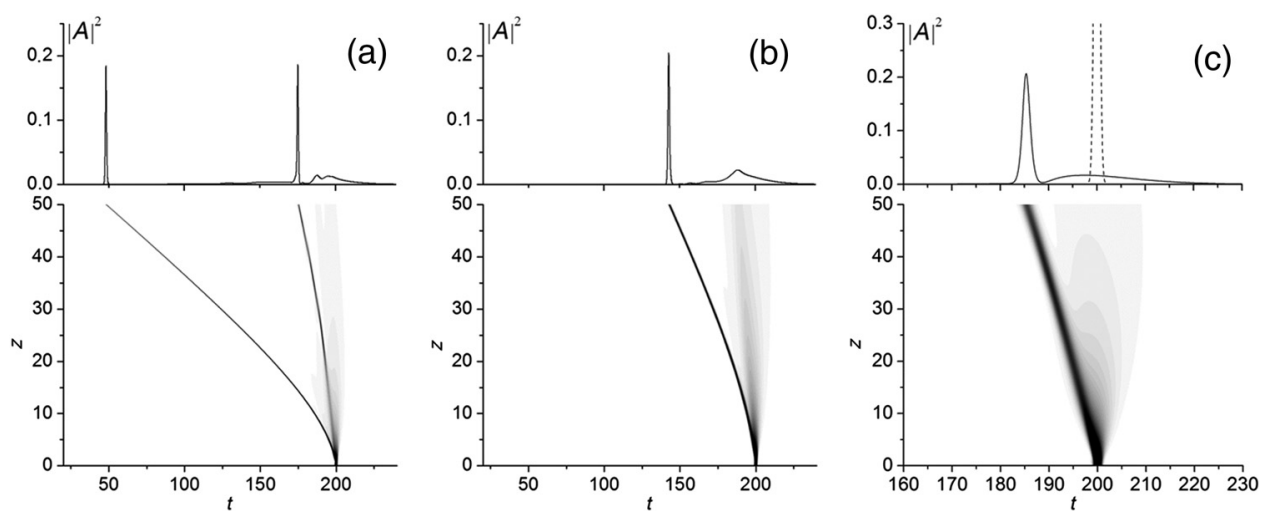

Fig. 10 Maximal intensity position for both subpulses at the incident Gaussian pulse propagation for the positive phase amplitude grating (a) $\xi=5$, (b) 2.5 , (c) 1 and $\delta_{0}=0.05, \tilde{\delta}=5$. The top figures show subpulse shapes at section $z=50$. Dashed line in top figure (c) shows the incident pulse shape.

response resonance, and for $D=0.1$. Consequently, we consider the dependence $f(\varepsilon)$ in Eq. (5). Strong nonlinear dependence $f(\varepsilon)$ results in rapid intensity decreasing and complicated scenario of multiple subpulses appearance. Nevertheless, the physical mechanism of the subpulses transformation into solitons and light acceleration, described in Sec. 4.1.1, remains the same.

Figures 11-13 show the pulse center shifting, the maximal intensity evolution, and multiple subpulses formation for the following sets of parameters characterizing the laser pulse energy depletion and phase grating strength, respectively, $\delta_{0}=0.05,0.1, \xi=2.5,1.25$. The frequency detuning for these parameters is very realistic, $\theta=50,25,12.5$, corresponding to the frequency detuning of $\approx 0.2 \omega_{p}, \approx 0.1 \omega_{p}$, and $\approx 0.05 \omega_{p}$, respectively, for $\omega_{p} \approx 2360 \mathrm{THz}(\lambda=800 \mathrm{~nm})$.

Due to the positive phase grating, induced by laser radiation and slow waves absorption for nanorods reshaping, the pulse center shifts into the area of time decreasing for all considered parameters [Fig. 11(a)]. We see in Figs. 11(b) and 12 that at the initial stage of the laser pulse propagation, its intensity strongly decreases as a result of laser energy absorption. This decreasing is even stronger than it takes place for the pulse dispersion spreading in a linear medium [dashed line in Fig. 11(b)]. Moreover, for the previous dependence $f(\varepsilon)$, the pulse intensity decreasing was less than it is for the pulse propagation in a linear medium. And even the pulse intensity increased partly at some sections of the medium [compare Figs. 9(b) and 11(b)]. Therefore, in the case under analysis, an influence of the amplitude grating on the maximal intensity evolution dominates until the propagation distance $z \leq 15$ [see Fig. 11(b)] at which the solitons appearance takes place.

Strong nonlinear dependence of laser energy absorption on the nanorod aspect ratio results in a multiple splitting of the incident pulse. As a result, a number of subpulses with soliton-like
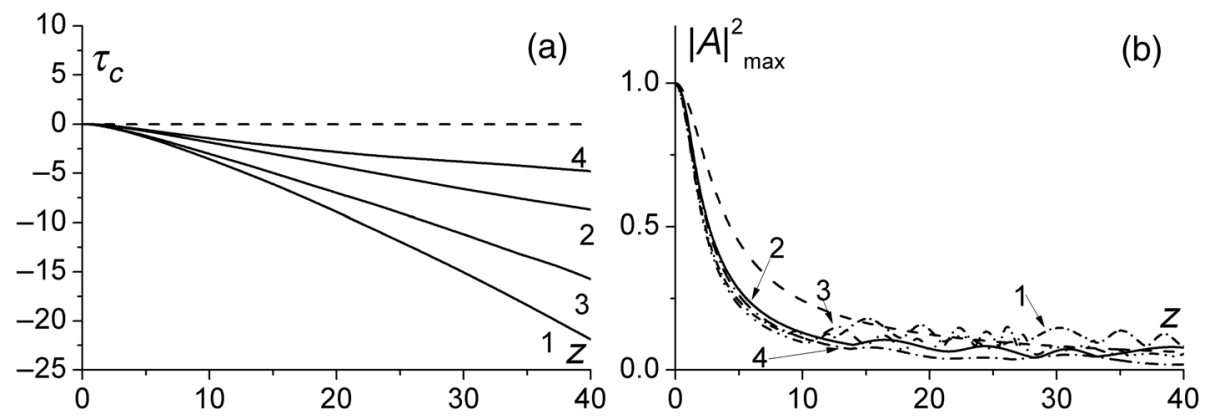

Fig. 11 (a) Pulse center shifting along the z-coordinate, calculated using Eq. (12) and (b) maximal intensity evolution for the positive phase amplitude grating and $\delta_{0}=0.05$ (curves 1 and 2), 0.1 (curves 3 and 4), $\xi=2.5$ (curves 1 and 3 ), 1.25 (curves 2 and 4 ), and $\tilde{\delta}=5$. Dashed lines correspond to the pulse propagation in a linear medium. 

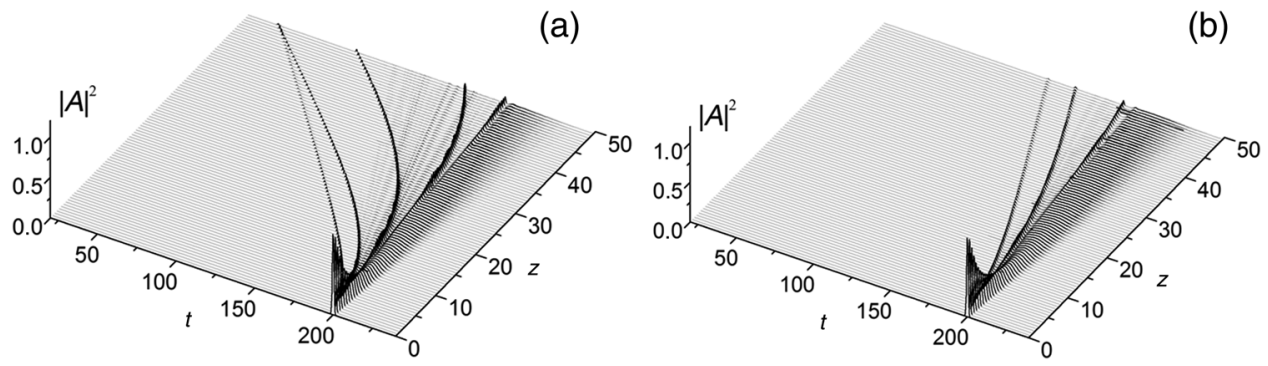

Fig. 12 Soliton formation at the incident Gaussian pulse propagation in the medium with parameters $\delta_{0}=0.05$, (a) $\xi=2.5$, (b) 1.25 , and $\tilde{\delta}=5$.
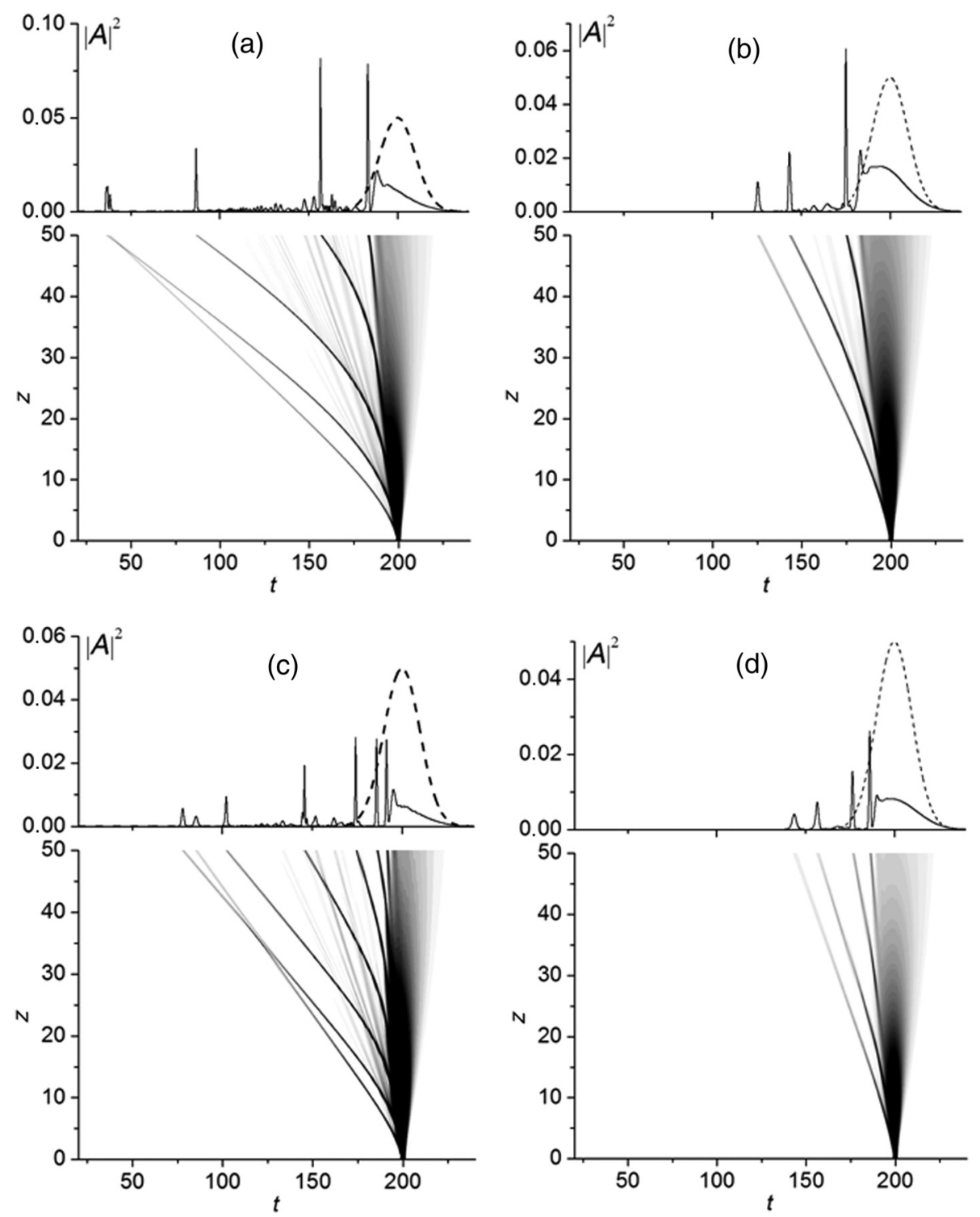

Fig. 13 Maximal intensity position for subpulses appearing at the incident Gaussian pulse propagation for (a) and (b) $\delta_{0}=0.05$, (c) and (d) 0.1 , (a) and (c) $\xi=2.5$, (b) and (d) 1.25 , and $\tilde{\delta}=5$. The top figures show subpulse shapes at section $z=50$. Dashed lines correspond to the pulse propagation in a linear medium. 
shape, trapped by the nanorod reshaping fronts, occur. Laser energy depletion gradually decreases the subpulse intensity, which leads to the subpulse velocity decreasing [see Eq. (44)]. So, the later appeared subpulse can travel through the earlier formed subpulse [Figs. 12(a), 13(a), and 13(c)].

Similar to the case of a linear dependence $f(\varepsilon)$ (far from response resonance on nanorod aspect ratio), a number of the subpulses and their velocity strongly depends on the phase grating strength for the dependence $f(\varepsilon)$ under consideration. So, the stronger is the grating strength, the faster is the subpulses, and the more subpulses are formed up to the section $z=50$ (Figs. 12 and 13). Remember that a stronger phase grating strength corresponds to a larger value of the pulse frequency detuning. So, the frequency detuning for Figs. 12(a) and 13(a) is about $0.2 \omega_{p}$, for $\omega_{p} \approx 2360 \mathrm{THz}(\lambda=800 \mathrm{~nm})$ while it is about $0.1 \omega_{p}$ for Figs. 12(b) and 13(b). The energy depletion also influences on the subpulses number as well as their intensity; the pulses intensity and their velocity decrease with energy depletion increasing and the subpulses number is growing [compare Figs. 13(a) and 13(c); Figs. 13(b) and 13(d)]. Larger values of energy depletion correspond to smaller values of the pulse frequency detuning, which is about $0.1 \omega_{p}$ for Fig. 13(c) and $0.05 \omega_{p}$ for Fig. 13(d). So, we can conclude that the pulse frequency detuning decrease results in the subpulses velocity decrease, whereas the number of subpulses depends on the phase grating strength and energy depletion. This demonstrates a fundamental role of nonlinear absorption and, as a consequence, of a pulse chirp formation for a soliton appearance.

One more important conclusion, following from comparison of Fig. 13, is that it is not necessary to realize high changing of the nanorods aspect ratio in time-area of the soliton formation. In particular, the nanorods aspect ratio changing in the whole time-area of the pulse does not exceed 0.4 (from 2.6 to 2.2) for all cases shown in Fig. 13, and it achieves its minimal value 0.15 (from 2.6 to 2.45) for the smallest frequency detuning [Fig. 13(d)]. The aspect ratio changing in the time-area of each soliton is less than 0.1. For example, for the case shown in Fig. 13(b), the aspect ratio changing is 0.02 (2.6 to 2.58), 0.04 (2.58 to 2.54), and 0.02 (2.54 to 2.4) in the timearea of the soliton, starting from the fastest to the slowest. This small aspect changing is very important for observation of such phenomenon.

\section{Conclusions}

For various parameters of laser pulse propagation in a medium with nanorods, including various detuning, we investigated acceleration of light (fast light) in comparison with light propagation in a linear medium. We considered a linear dependence of laser energy absorption on nanorod aspect ratio and a strong nonlinear dependence in the vicinity of the maximum of this dependence. Acceleration of light can be accompanied by the pulse splitting for the linear dependence of laser energy absorption dependence on the nanorod aspect ratio, and a multiple splitting can take place for a laser pulse interaction with nanorods near the nonlinear absorption resonance. In all considered cases, acceleration of light is accompanied by soliton formation.

The physical reason for laser pulse acceleration is the pulse chirping due to the induced phase and amplitude gratings and nanorods reshaping, which leads to nonstationary interaction of laser radiation with the medium and time-dependent changing of nanorod aspect ratio. As a result, a soliton-like propagation can take place due to the trapping of laser radiation by nanorod reshaping fronts.

It is very important to stress that the soliton propagation is accompanied by the minor aspect ratio changing if the soliton formation occurs for a laser pulse interaction with nanorods near the nonlinear absorption resonance. This is very important for observation of such phenomenon in practice because such minor shape transformation takes less time for its realization.

We confirmed our computer results by developing an approximate analytical soliton taking into account SOD of a medium. This allows us to derive analytical expressions for the soliton amplitude, duration, and phase evolution, and predict existence of two new types of solitons-an accelerating chirped soliton and a slowing down chirped soliton - for the same parameters set.

We also developed the analytical solution of the considered problem in the framework of nonlinear geometric optics approximation. This analysis allowed revealing the role of the pulse chirp in the light acceleration. In particular, we demonstrated the pulse center shift into the area of time decreasing if a positive phase grating is induced by laser radiation. 


\section{Appendix}

Here, we give a derivation of equations for an approximate soliton-like solution for the pulse propagation in a medium with TPA and nanorods for $f(\varepsilon)$ in Eq. (4). Representing the complex amplitude $A(z, t)$ in a soliton-like form [Eq. (25)] and substituting it into Eqs. (9)-(10), we get the equations with respect to amplitude and phase distribution evolution of the soliton

$$
\begin{gathered}
\frac{\mathrm{d} \ln B}{\mathrm{~d} z}+t h \zeta\left(\zeta \frac{\mathrm{d} \ln \tau_{s}}{\mathrm{~d} z}+\frac{1}{\tau_{s}} \frac{\mathrm{d} t_{c}}{\mathrm{~d} z}\right)+\frac{D}{\tau_{s}^{2}}\left(\frac{\partial^{2} s}{\partial \zeta^{2}}-2 t h \zeta \frac{\partial s}{\partial \zeta}\right)+\delta_{0} f B^{2}\left(1-t h^{2} \zeta\right)=0 \\
\frac{\partial s}{\partial z}-\frac{\partial s}{\partial \zeta}\left(\zeta \frac{\mathrm{d} \ln \tau_{s}}{\mathrm{~d} z}+\frac{1}{\tau_{s}} \frac{\mathrm{d} t_{c}}{\mathrm{~d} z}\right)-\frac{D}{\tau_{s}^{2}}\left(2 t h^{2} \zeta-1\right)+\frac{D}{\tau_{s}^{2}}\left(\frac{\partial s}{\partial \zeta}\right)^{2}-\xi f B^{2}\left(1-t h^{2} \zeta\right)=0, \\
\frac{1}{\tau_{s}} \frac{\partial f}{\partial \zeta}=-\tilde{\delta} f B^{4} c h^{-4} \zeta .
\end{gathered}
$$

Obviously, the last equation can be integrated

$$
f(z, \zeta)=f_{0} \exp \left[-\tilde{\delta} B^{4} \tau_{s}\left(t h \zeta-1 / 3 t h^{3} \zeta+2 / 3\right)\right]
$$

To find a chirped soliton, we represent the pulse phase $s(t, \zeta)$ as in Eq. (27). Then, substituting Eqs. (27) and (51) in Eqs. (48) and (49), we get the following equations:

$$
\begin{gathered}
\frac{\mathrm{d} \ln B}{\mathrm{~d} z}+t h \zeta\left(\zeta \frac{\mathrm{d} \ln \tau_{s}}{\mathrm{~d} z}+\frac{1}{\tau_{s}} \frac{\mathrm{d} t_{c}}{\mathrm{~d} z}\right)+\frac{D}{\tau_{s}^{2}}\left[h\left(1-3 t h^{2} \zeta\right)-2 b t h \zeta\right] \\
+\delta_{0} f_{0} B^{2}\left(1-t h^{2} \zeta\right) \exp \left[-\tilde{\delta} B^{4} \tau_{s}\left(t h \zeta-1 / 3 t h^{3} \zeta+2 / 3\right)\right]=0 \\
\frac{\mathrm{d} a}{\mathrm{~d} z}+\frac{\mathrm{d} b}{\mathrm{~d} z} \zeta+\frac{\mathrm{d} h}{\mathrm{~d} z} \ln c h \zeta-(b+h \cdot t h \zeta)\left(\zeta \frac{\mathrm{d} \ln \tau_{s}}{\mathrm{~d} z}+\frac{1}{\tau_{s}} \frac{\mathrm{d} t_{c}}{\mathrm{~d} z}\right)+\frac{D}{\tau_{s}^{2}}\left[(b+h \cdot t h \zeta)^{2}-2 t h^{2} \zeta+1\right] \\
-\xi f_{0} B^{2}\left(1-t h^{2} \zeta\right) \exp \left[-\tilde{\delta} B^{4} \tau_{s}\left(t h \zeta-1 / 3 t h^{3} \zeta+2 / 3\right)\right]=0 .
\end{gathered}
$$

The structure of the above equations is the following: the first four terms in Eq. (52) can be represented as a linear combination of $t h \zeta, t h^{2} \zeta, \zeta t h \zeta$, and zero power of $t h \zeta$, while the first six terms in Eq. (53) can be represented as a linear combination of $\zeta, t h \zeta, t h^{2} \zeta, \zeta \operatorname{th} \zeta$, and zero power of $t h \zeta$. Let us note that, as it follows from Eq. (52), the nonlinear absorption causes the soliton amplitude damping. The influence of the pulse chirp depends on the medium dispersion, the pulse positive chirp $(h>0)$ in a medium with normal dispersion $(D>0$ in our notations) also causes the soliton amplitude damping due to the pulse spreading while the negative chirp $(h<0)$ causes amplitude increasing because of the pulse compression. The opposite dependence takes place in a medium with anomalous dispersion. The phase grating influences the pulse phase shift along the $z$-coordinate $a(z)$, it increases for a positive phase grating and decreases for its negative value [Eq. (53)]. Of course, this grating defines the pulse chirp in dependence on the dispersion of the medium.

To write the separate equations for each of the functions $a(z), b(z), h(z), \tau_{s}(z), t_{c}(z)$, and $B(z)$ in Eqs. (52) and (53), we first expand the exponent in Eqs. (52) and (53) into a power series with respect to $\tilde{\delta} B^{4} \tau_{s} t h \zeta$

$$
\begin{aligned}
& \left(1-t h^{2} \varsigma\right) \exp \left[-\tilde{\delta} B^{4} \tau_{s}\left(t h \zeta-1 / 3 t h^{3} \zeta+2 / 3\right)\right] \\
& \quad=\exp \left(-2 / 3 \tilde{\delta} B^{4} \tau_{s}\right)\left\{1-\tilde{\delta} B^{4} \tau_{s} t h \zeta+\left[1 / 2\left(\tilde{\delta} B^{4} \tau_{s}\right)^{2}-1\right] t h^{2} \zeta+\ldots\right\}
\end{aligned}
$$

We need to do this for the explicit definition of nonlinear absorption and refraction affect on the parameters of the pulse because the exponent in Eqs. (52) and (53) introduces an action in all functions changing along the $z$-coordinate. Then, we collect the coefficients for $t h \zeta, t h^{2} \zeta, \zeta \operatorname{th} \zeta$, and zero power of $t h \zeta$ in Eq. (52), and for this equation validity at each time moment, we require that these coefficients are equal to zero. As a result, one can write the following equations: 


$$
\begin{gathered}
\frac{\mathrm{d} \ln B}{\mathrm{~d} z}+\frac{D}{\tau_{s}^{2}} h+\delta_{0} f_{0} B^{2} \exp \left(-2 / 3 \tilde{\delta} B^{4} \tau_{s}\right)=0, \\
\frac{\mathrm{d} t_{c}}{\mathrm{~d} z}-2 \frac{D}{\tau_{s}} b-\delta_{0} f_{0} \tilde{\delta} \tau_{s}^{2} B^{6} \exp \left(-2 / 3 \tilde{\delta} B^{4} \tau_{s}\right)=0 \\
-3 D h+\delta_{0} f_{0} \tau_{s}^{2} B^{2} \exp \left(-2 / 3 \tilde{\delta} B^{4} \tau_{s}\right)\left[1 / 2\left(\tilde{\delta} B^{4} \tau_{s}\right)^{2}-1\right]=0, \\
\frac{\mathrm{d} \ln \tau_{s}}{\mathrm{~d} z}=0 .
\end{gathered}
$$

A few words about the terms in Eq. (54). The second term in Eq. (54) describes the soliton amplitude changing due to the mutual action of pulse chirp and SOD, whereas the third term is due to the nonlinear absorption. Obviously, the nonlinear absorption causes the soliton amplitude damping. However, the pulse chirp can result in the soliton amplitude growing or decreasing depending on the sign of the product $D h$. So, in the medium with normal dispersion ( $D>0$ in our notations), a positive chirp causes the soliton amplitude decreasing while a negative chirp causes the pulse intensity growing. The opposite situation occurs in the medium with anomalous dispersion. Nevertheless, as we will see below, competition of these two mechanisms results in the soliton intensity oscillations on certain propagation distance and its further damping along the $z$-coordinate. It is essential to stress that the chirp of the appearing soliton is determined by the soliton amplitude [Eq. (56)], if the expression $1 / 2\left(\tilde{\delta} B^{4} \tau_{s}\right)^{2}-1$ is positive then the chirp will be also positive, for the opposite case its sign will be negative. We see that a pulse chirp appearance $(h \neq 0)$ is a consequence of the medium nonlinear absorption $\left(\delta_{0} \neq 0\right)$.

Due to a pulse chirp appearance because of the nonlinear absorption, the pulse center always shifts into the area of increasing time. Action of SOD on the pulse center shifting depends on the product $D b$ sign. If a caring frequency decreases then the pulse center can shift in the area of time increasing. For the opposite case, the pulse center can shift in the area of decreasing time. In a general case, oscillations of the pulse center can take place. Equation (57) implies the constant value of the soliton duration along the $z$-coordinate $\tau_{s}(z)=\tau_{s 0}$. It is the consequence of the uniqueness of the term with $\zeta$ th $\zeta$ in Eq. (52). It should be emphasized that in order to get the corresponding evolution of this pulse characteristic along the $z$-coordinate it is necessary to take into account an additional term in the phase representation Eq. (27).

For writing the equations with respect to functions $a(z), b(z), h(z)$ from Eq. (53), we do the same steps as we made above. As a result, we get the following equations:

$$
\begin{gathered}
\frac{\mathrm{d} a}{\mathrm{~d} z}-b \frac{1}{\tau_{s}} \frac{\mathrm{d} t_{c}}{\mathrm{~d} z}+\frac{D}{\tau_{s}^{2}}\left(1+b^{2}\right)-\xi f_{0} B^{2} \exp \left(-2 / 3 \tilde{\delta} B^{4} \tau_{s}\right)=0, \\
\frac{\mathrm{d} b}{\mathrm{~d} z}-h \cdot \frac{1}{\tau_{s}} \frac{\mathrm{d} t_{c}}{\mathrm{~d} z}+2 \frac{D}{\tau_{s}^{2}} \cdot b \cdot h+\xi f_{0} \tilde{\delta} \tau_{s} B^{6} \exp \left(-2 / 3 \tilde{\delta} B^{4} \tau_{s}\right)=0, \\
D\left(h^{2}-2\right)+\xi f_{0} \tau_{s}^{2} B^{2} \exp \left(-2 / 3 \tilde{\delta} B^{4} \tau_{s}\right)\left[1-1 / 2\left(\tilde{\delta} B^{4} \tau_{s}\right)^{2}\right]=0 .
\end{gathered}
$$

Equation (60) together with Eq. (56) determines the pulse chirp in dependence of nonlinear absorption $\left(\delta_{0}, \tilde{\delta}\right)$, phase grating $(\xi)$, and the medium dispersion $(D)$. One has to emphasize that Eqs. (54)-(57) and Eqs. (58)-(60) must be valid simultaneously, it means that they should be consistent. This requirement may be satisfied. Indeed, as it was already mentioned, the inequality $1 / 2\left(\tilde{\delta} B^{4} \tau_{s}\right)^{2}-1>0$ follows from Eq. (56) for a positive product $D h$. Equation (60) gives for this case the inequality $D\left(h^{2}-2\right)>0$, which implies that $h^{2}>2$ for a medium with normal dispersion and positive self-action parameter $\xi$, for example. Therefore, these two inequalities can be satisfied simultaneously.

Equation (59) together with Eq. (55) determines the pulse frequency shift along the $z$-coordinate and they must be valid simultaneously. This is possible, obviously, if the pulse chirp is absent $(h=0)$, for example, then the pulse frequency shift decreases for the positive $\xi$ and increases for its negative value. 


\section{Acknowledgments}

The investigation was made with the support of the Russian Science Foundation (Grant No. 1421-00081).

\section{References}

1. P. Zijlstra, J. W. M. Chon, and M. Gu, "Five-dimensional optical recording mediated by surface plasmons in gold nanorods," Nature 459, 410-413 (2009).

2. H. Ditlbacher et al., "Spectrally coded optical data storage by metal nanoparticles," Opt. Lett. 25, 563-565 (2000).

3. O. Wilson, G. J. Wilson, and P. Mulvaney, "Laser writing in polarized silver nanorod films," Adv. Mater. 14, 1000-1004 (2002).

4. J. Pérez-Juste et al., "Optical control and patterning of gold-nanorod-poly (vinyl alcohol) nanocomposite films," Adv. Funct. Mater. 15, 1065-1071 (2005).

5. A. B. Taylor, J. Kim, and J. W. M. Chon, "Detuned surface plasmon resonance scattering of gold nanorods for continuous wave multilayered optical recording and readout," Opt. Express 20(5), 5069-5081 (2012).

6. B. J. Roxworthy et al., "Multifunctional plasmonic film for recording near-field optical intensity," Nano Lett. 14, 4687-4693 (2014).

7. H. Long et al., "Multilayer $\mathrm{Au} / \mathrm{TiO}_{2}$ composite films with ultrafast third-order nonlinear optical properties," Chin. Phys. Lett. 25(11), 4135-4138 (2008).

8. A. L. Stepanov et al., "Nonlinear optical properties of gold nanoparticles synthesized by ion implantation in sapphire matrix," Tech. Phys. Lett. 31(8), 702-705 (2005).

9. D. Faccio et al., "Measurement of the third-order nonlinear susceptibility of Ag nanoparticles in glass in a wide spectral range," Europhys. Lett. 43(2), 213-218 (1998).

10. V. P. Drachev et al., "Size dependent $\chi^{3}$ for conduction electrons in Ag nanoparticles," Nano Lett. 4(8), 1535-1539 (2004).

11. X. C. Yang et al., "Optical nonlinearity and ultrafast dynamics of ion exchanged silver nanoparticles embedded in soda-lime silicate glass," Chin. Sci. Bull. 53(5), 695-699 (2008).

12. S.-M. Ma et al., "Cubic nonlinear optical properties of Ag nanoparticles and $\mathrm{Ag} / \mathrm{Au}$ coreshells," J. Phys. Conf. Ser. 109, 012024 (2008).

13. N. N. Lepeshkin et al., "Enhanced nonlinear optical response of one-dimensional metaldielectric photonic crystals," Phys. Rev. Lett. 93(12), 123902 (2004).

14. N. Del Fatti, D. Christofilos, and F. Vallée, "Optical response of a single gold nanoparticle," Gold Bull. 41(2), 147-158 (2008).

15. C. M. J. Wijers et al., "Optical response of layers of embedded semiconductor quantum dots," Phys. Rev. B 74, 035323 (2006).

16. A. Mohammadi, V. Sandoghdar, and M. Agio, "Gold nanorods and nanospheroids for enhancing spontaneous emission," New J. Phys. 10, 105015 (2008).

17. R. Jin et al., "Correlating second harmonic optical responses of single Ag nanoparticles with morphology," J. Am. Chem. Soc. 127, 12482 (2005).

18. S. Eustis and M. A. El-Sayed, "Why gold nanoparticles are more precious than pretty gold: noble metal surface plasmon resonance and its enhancement of the radiative and nonradiative properties of nanocrystals of different shapes," Chem. Soc. Rev. 35, 209-217 (2006).

19. A. B. Taylor, A. M. Siddiquee, and J. W. M. Chon, "Below melting point photothermal reshaping of single gold nanorods driven by surface diffusion," ACS Nano 8, 12071 (2014).

20. O. Gülseren, F. Ercolessi, and E. Tosatti, "Premelting of thin wires," Phys. Rev. B 51, 73777380 (1995).

21. A. Hoss et al., "Roughening and melting of Au(110) surfaces," Phys. Rev. B 45, 8714-8720 (1992).

22. P. Buffat and J. P. Borel, "Size effect on the melting temperature of gold particles," Phys. Rev. A 13, 2287-2298 (1976).

23. H. Reiss, P. Mirabel, and R. L. Whetten, "Capillarity theory for the 'coexistence' of liquid and solid clusters," J. Phys. Chem. 92, 7241-7246 (1988)

24. H. Sakai, "Surface-induced melting of small particles," Surf. Sci. 351, 285-291 (1996). 
Trofimov and Lysak: Superluminality phenomenon at a femtosecond laser pulse propagation...

25. G. K. Goswami and K. K. Nanda, "Size-dependent melting of finite-length nanowires," J. Phys. Chem. C 114(34), 14327 (2010).

26. A. Blanco-Redondo et al., "Observation of soliton compression in silicon photonic crystals," Nat. Commun. 5, 3160 (2014).

27. C. A. Husko et al., "Soliton dynamics in the multiphoton plasma regime," Sci. Rep. 3, 1-8 (2013).

28. F. Raineri et al., "Time-domain mapping of nonlinear pulse propagation in photonic-crystal slow-light waveguides," Phys. Rev. Lett. A 87, 041802 (2013).

29. A. Peacock, "Soliton propagation in tapered silicon core fibres," Opt. Lett. 35, 3697-3699 (2010).

30. W. Ding et al., "Time and frequency domain measurements of solitons in subwavelength silicon waveguides using a cross-correlation technique," Opt. Express 18, 26625 (2010).

31. J. Zhang et al., "Optical solitons in a silicon waveguide," Opt. Express 15, 7682-7688 (2007).

32. A. Biswas et al., "Dispersive optical solitons by the semi-inverse variational principle," J. Mod. Opt. 59(3), 213-217 (2012).

33. R. Kohl et al., "Optical soliton perturbation in a non-Kerr law media," Opt. Laser Technol. 40, 647-662 (2008).

34. A. Biswas et al., "Optical soliton perturbation in a non-Kerr law media: traveling wave solution," Opt. Laser Technol. 44, 263-268 (2012).

35. V. A. Trofimov, T. M. Lysak, and S. Lan, "Influence of ellipticity of nanorods on both TPA of femtosecond laser pulse and transformation of pulse spectrum," Proc. SPIE 8424, 842435 (2012).

36. V. A. Trofimov, T. M. Lysak, and S. Lan, "Influence of absorption spectrum width of nanorods on TPA of femtosecond laser pulse," Proc. SPIE 8463, 84630J (2012).

37. V. A. Trofimov and T. M. Lysak, "Acceleration of light and soliton formation due to nonlinear absorption of femtosecond laser pulse energy by the medium containing nanorods," J. Opt. Soc. Am. B 33(1), 62-74 (2016).

38. V. A. Trofimov and T. M. Lysak, "Self-similar mode of laser pulse propagation in a medium containing nanorods," J. Opt. 18(1), 025501 (2016).

39. V. A. Trofimov, O. V. Matusevich, and D. A. Smotrov, "Mode of propagation of optical radiation with self-similar pulse shape in layered medium with nonlinear absorption," Proc. SPIE 8095, 80951K (2011).

40. V. A. Trofimov, T. M. Lysak, and I. G. Zakharova, "2D self-similar profile for laser beam propagation in medium with saturating multi-photon absorption," J. Phys. Conf. Ser. 691(1), 012013 (2016).

41. S. Liberati, S. Sonego, and M. Visser, "Faster-than-c signals, special relativity, and causality," Ann. Phys. 298(1), 167-185 (2002).

42. A. Haché and L. Poirier, "Anomalous dispersion and superluminal group velocity in a coaxial photonic crystal: theory and experiment," Phys. Rev. E. 65(3), 036608 (2002).

43. G. M. Shore, "Superluminality and UV completion," Nucl. Phys. B 778(3), 219-258 (2007).

44. G. Nimtz, "Superluminal signaling by photonic tunneling," IEEE J. Sel. Top. Quantum Electron. 9(1), 79-85 (2003).

45. G. Nimtz, "On superluminal tunneling," Prog. Quantum Electron. 27(6), 417-450 (2003).

46. I. Blonskyi et al., "Manifestations of sub- and superluminality in filamented femtosecond laser pulse in fused silica," Opt. Commun. 282(9), 1913-1917 (2009).

47. V. S. Butylkin et al., Resonant Nonlinear Interactions of Light with Matter, Springer, Berlin (1989).

48. H.-D. Deng, "Role of interfering optical fields in the trapping and melting of gold nanorods and related clusters," Opt. Express 20, 10963 (2012).

49. G. T. Boyd, Z. H. Yu, and Y. R. Shen, "Photoinduced luminescence from the noble-metals and its enhancement on roughened surfaces," Phys. Rev. B 33(12), 7923-7936 (1986).

50. S. Link and M. A. El-Sayed, "Simulation of the optical absorption spectra of gold nanorods as a function of their aspect ratio and the effect of the medium dielectric constant," J. Phys. Chem. B 109(20), 10531 (2005). 
Trofimov and Lysak: Superluminality phenomenon at a femtosecond laser pulse propagation...

51. S. Link, M. B. Mohamed, and M. A. El-Sayed, "Simulation of the optical absorption spectra of gold nanorods as a function of their aspect ratio and the effect of the medium dielectric constant," J. Phys. Chem. B 103(16), 3073-3077, (1999).

52. M. N. Polyanskiy, "Refractive index database," https://refractiveindex.info (18 April 2017).

53. K. Imura, T. Nagahara, and H. Okamoto, "Near-field two-photon-induced photoluminescence from single gold nanorods and imaging of plasmon modes," J. Phys. Chem. B 109(27), 13214 (2005).

54. G. P. Agrawal, Nonlinear Fiber Optics, Academic Press, Boston (1989).

55. J. Rauch, "Hyperbolic partial differential equations and geometric optics," in Graduate Studies in Mathematics, D. Cox et al., Eds., Vol. 133, p. 364, American Mathematical Society, Providence (2012).

Vyacheslav A. Trofimov is a professor of the chair of computational methods and the head of the Laboratory of Mathematical Modeling in Physics at Lomonosov Moscow State University. He graduated with honors in 1980, obtained his PhD in laser physics in 1983 and his degree of doctor of science in 1995 from Lomonosov Moscow State University. His main field research experience includes laser physics, computer simulation, mathematical modeling, finite-difference scheme, and programming, including multiprocessor computers.

Tatiana M. Lysak is a senior scientific researcher at the Laboratory of Mathematical Modeling in physics at Lomonosov Moscow State University. She graduated with honors from Lomonosov Moscow State University (Physical Department) in 1985 and obtained her PhD in calculus mathematics from the same university in 1990. Her current research interests include interaction of laser light with matter, frequency conversion of femtosecond laser pulses, optical bistability, and optical solitons. 